\title{
Smooth and fast versus instantaneous quenches in quantum field theory
}

\author{
Sumit R. Das, ${ }^{a}$ Damián A. Galante ${ }^{b, c, d}$ and Robert C. Myers ${ }^{c}$ \\ ${ }^{a}$ Department of Physics and Astronomy, University of Kentucky, \\ 505 Rose St, Lexington, KY 40506, U.S.A. \\ ${ }^{b}$ Department of Applied Mathematics, University of Western Ontario, \\ 1151 Richmond Street, London, ON N6A 5B7, Canada \\ ${ }^{c}$ Perimeter Institute for Theoretical Physics, \\ 55 Caroline St N, Waterloo, ON N2L 2Y5, Canada \\ ${ }^{d}$ Kavli Institute for Theoretical Physics, University of California, \\ Kohn Hall, Santa Barbara, CA 93106, U.S.A. \\ E-mail: das@pa.uky.edu, dgalante@perimeterinstitute.ca, \\ rmyers@perimeterinstitute.ca
}

ABSTRACT: We examine in detail the relationship between smooth fast quantum quenches, characterized by a time scale $\delta t$, and instantaneous quenches, within the framework of exactly solvable mass quenches in free scalar field theory. Our earlier studies [1,2] highlighted that the two protocols remain distinct in the limit $\delta t \rightarrow 0$ because of the relation of the quench rate to the UV cut-off, i.e., $1 / \delta t \ll \Lambda$ always holds in the fast smooth quenches while $1 / \delta t \sim \Lambda$ for instantaneous quenches. Here we study UV finite quantities like correlators at finite spatial distances and the excess energy produced above the final ground state energy. We show that at late times and large distances (compared to the quench time scale) the smooth quench correlator approaches that for the instantaneous quench. At early times, we find that for small spatial separation and small $\delta t$, the correlator scales universally with $\delta t$, exactly as in the scaling of renormalized one point functions found in earlier work. At larger separation, the dependence on $\delta t$ drops out. The excess energy density is finite (for finite $m \delta t$ ) and scales in a universal fashion for all $d$. However, the scaling behaviour produces a divergent result in the limit $m \delta t \rightarrow 0$ for $d \geq 4$, just as in an instantaneous quench, where it is UV divergent for $d \geq 4$. We argue that similar results hold for arbitrary interacting theories: the excess energy density produced is expected to diverge for scaling dimensions $\Delta>d / 2$.

KEYwords: AdS-CFT Correspondence, Effective field theories, Holography and condensed matter physics (AdS/CMT)

ARXiv EPrint: 1505.05224 


\section{Contents}

1 Introduction 1

2 Bogoliubov coefficients for smooth and instantaneous quenches 3

3 Late time spatial correlators $\quad 6$

3.1 Numerical results for various dimensions 8

$\begin{array}{lll}3.2 & \text { Small } r \text { behavior and counterterms for local operators } & 12\end{array}$

4 Universal scaling in quenched spatial correlators $\quad 15$

5 Late time behaviour of $\phi^{2} \quad 17$

$\begin{array}{ll}5.1 \text { Review of } d=3 & 17\end{array}$

$\begin{array}{ll}5.2 \text { Higher dimensions } & 20\end{array}$

$\begin{array}{lll}5.3 & \text { Regulated instantaneous quench } & 23\end{array}$

$6 \quad$ The energy density at late times $\quad 26$

$\begin{array}{lll}7 & \text { Excess energy for general theories } & 29\end{array}$

8 Discussion $\quad 31$

$\begin{array}{ll}\text { A Review of constant mass correlators } & 38\end{array}$

\section{Introduction}

Universal scaling behavior in systems undergoing a quantum (or thermal) quench which involves critical points have been a subject of great interest in recent years [7-9]. ${ }^{1}$ The classic example of such behavior is Kibble-Zurek scaling [7, 8] which involves a quench which starts from a gapped phase at a rate which is slow compared to the scale set by the initial gap. At the other extreme, there are a different set of universal behaviors in twodimensional field theories which are quenched instantaneously from a gapped phase to a critical point [10-12] and for instantaneous quenches which can be treated perturbatively. ${ }^{2}$

The AdS/CFT correspondence has yielded important insight in this area, both for Kibble-Zurek scaling [14-19] and for novel non-equilibrium phases [20-22]. Perhaps more significantly, holographic studies have led to the discovery of new scaling behavior for smooth quenches which are fast compared to the physical mass scales, but slow compared to the UV scale [23-25]. In [1] and [2] we argued that this scaling law holds regardless

\footnotetext{
${ }^{1}$ For example see the following reviews [3-6].

${ }^{2}$ For a review and further references see [13].
} 
of holography, and is valid for time dependent relevant deformations of generic conformal field theories (see also [26]). Consider an action

$$
S=S_{C F T}+\int d^{d} x \lambda(t) \mathcal{O}_{\Delta}(\vec{x}, t)
$$

where the conformal dimension of the operator $\mathcal{O}$ is $\Delta$ and $\lambda(t)$ interpolates between the constant values $\lambda_{1}$ and $\lambda_{2}$ (with an amplitude variation of $\delta \lambda$ ) over a time of order $\delta t$. Then in the fast quench limit

$$
\delta t \ll \lambda_{1}^{1 /(\Delta-d)}, \lambda_{2}^{1 /(\Delta-d)}, \delta \lambda^{1 /(\Delta-d)}
$$

the renormalized energy density $\delta \mathcal{E}_{\text {ren }}$ scales as

$$
\delta \mathcal{E}_{\mathrm{ren}} \sim \frac{\delta \lambda^{2}}{\delta t^{2 \Delta-d}} .
$$

Similarly, the peak of the renormalized expectation value of the quenched operator, measured at times earlier than or soon after the end of the quench, was also found to scale as

$$
\left\langle\mathcal{O}_{\Delta}\right\rangle_{\text {ren }} \sim \frac{\delta \lambda}{\delta t^{2 \Delta-d}},
$$

This general result emerged out of detailed investigations of exactly solvable mass quenches in free bosonic and fermionic theories. One important outcome of our analysis was an understanding of the relationship between smooth fast quenches for small $\delta t$ and the instantaneous quenches of e.g., [10-12]. The latter involve a quench rate which is fast compared to all scales, including the UV cutoff, while smooth quench rates are faster than any physical scales, but slower than the cutoff scale. On the other hand, local quantities like the energy density or $\langle\mathcal{O}\rangle$ involve a sum over all momenta all the way to the cutoff - for such quantities one does not expect the smooth quench result to agree with those in the instantaneous quench. By the same token, one would expect that for correlators at finite separations larger than $\delta t$, there should be agreement. In $[1,2]$ we also explored if the late time behavior of local quantities also agree, finding agreement at least in the $d=3$ case.

In this paper we continue to explore the relationship between fast but smooth quenches and instantaneous quenches in further detail. Our analysis will focus on quenches in free scalar field theory with a time-dependent mass. However we argue that the lessons we draw there will be valid for quenches in interacting theories of the type described above.

The scaling laws in $[1,2]$ were derived for renormalized composite operators, which are the appropriate quantities for quench rates much slower than the cutoff scale. In this work, we examine the late time behavior of such operators. In addition, we focus on quantities which are UV finite, e.g., two-point correlation functions at finite spatial separations and the excess energy over the ground state energy at late times.

In section 3 , we consider late time correlators, $t \gg \delta t$. We will show that for (suitably defined) large spatial separations, these correlators agree with the correlators for an instantaneous quench. For separations $r$ which are very small, i.e., $m r \ll 1$ there is once again agreement, reflecting the fact that the dominant singular behavior for small separation is independent of any time dependence of the mass. The corrections to this leading 
small distance behavior are in one-to-one correspondence with the counterterms necessary to renormalize the composite operator $\phi^{2}$. In particular, the subleading small distance divergences involve derivatives of the mass function for $d \geq 6$. For intermediate separations, the two quench protocols lead to genuinely different results.

In section 4 , we turn our attention to correlators at finite times $t \sim \delta t$ and show that for $r \delta t \gg 1$ the correlator becomes independent of $\delta t$ as expected. For $m^{-1}>\delta t>r$ we find that the correlator exhibits a scaling behavior identical to that of the renormalized local operator $\left\langle\phi^{2}\right\rangle$.

In section 5, we consider the renormalized local quantity $\left\langle\phi^{2}\right\rangle$ at late times. We show that this quantity agrees for both quench protocols only for $d=3$. For $d=5$ and finite $\delta t$, there is a slight difference between the smooth and the instantaneous answer in the limit of $\delta t \rightarrow 0$, while for the instantaneous quench, $\left\langle\phi^{2}\right\rangle$ is UV divergent for $d>5$.

In section 6 , we consider the difference of the energy at late times and the ground state energy with the final value of the mass. This is one measure of the excess energy produced during the quench. We show that this quantity is explicitly UV finite. For $d \leq 3$ this becomes independent of $\delta t$, in the $m \delta t \ll 1$ limit. The next order correction, which scales as a power of $\delta t$, is identical to the behavior of the renormalized energy in [1,2]. For $d \geq 4$, the energy diverges in the $\delta t \rightarrow 0$ limit, in the same way as the renormalized energy considered in $[1,2]$.

In section 7, we discuss the validity of our results for the excess energy produced for general interacting field theories.

In section 8 , we conclude with a brief discussion of our results and also consider various possible measures of the energy produced by the quench and their relationship.

\section{Bogoliubov coefficients for smooth and instantaneous quenches}

Consider a scalar field in $d$ space-time dimensions with a time dependent mass,

$$
S=-\int d t \int d^{d-1} x \frac{1}{2}\left[(\partial \phi)^{2}+m^{2}(t) \phi^{2}\right] .
$$

This theory is exactly solvable for a variety of different mass profiles, as described in $[1,2]$. The quench protocol which we focus on here, involves the mass going from an initial value $m$ to zero at late times over a time scale $\delta t$ with the smooth profile

$$
m^{2}(t)=m^{2}\left(\frac{1-\tanh (t / \delta t)}{2}\right) .
$$

To solve the Klein-Gordon equation, we decompose the scalar field into momentum modes

$$
\phi=\int \frac{d^{d-1} k}{(2 \pi)^{(d-1) / 2}}\left(a_{\vec{k}} u_{\vec{k}}+a_{\vec{k}}^{\dagger} u_{\vec{k}}^{*}\right), \quad \text { where } \quad\left[a_{\vec{k}}, a_{\vec{k}^{\prime}}^{\dagger}\right]=\delta^{d-1}\left(\vec{k}-\vec{k}^{\prime}\right) .
$$

The exact solution of the field equation is given by $[1,2,27]$

$$
\begin{aligned}
u_{\vec{k}}=\frac{1}{\sqrt{2 \omega_{\text {in }}}} & \exp \left(i \vec{k} \cdot \vec{x}-i \omega_{+} t-i \omega_{-} \delta t \log (2 \cosh t / \delta t)\right) \\
& \times{ }_{2} F_{1}\left(1+i \omega_{-} \delta t, i \omega_{-} \delta t ; 1-i \omega_{\text {in }} \delta t ; \frac{1+\tanh (t / \delta t)}{2}\right),
\end{aligned}
$$


where ${ }_{2} F_{1}$ is the usual hypergeometric function and

$$
\begin{aligned}
& \omega_{\text {in }}=\sqrt{\vec{k}^{2}+m^{2}}, \quad \omega_{\text {out }}=|\vec{k}|, \\
& \omega_{ \pm}=\left(\omega_{\text {out }} \pm \omega_{\text {in }}\right) / 2 .
\end{aligned}
$$

The modes $u_{\vec{k}}$ are the "in" modes: they behave as plane waves in the infinite past and the $a_{\vec{k}}$ annihilate the in-vacuum, i.e., $a_{\vec{k}}|i n, 0\rangle=0$. There is also another set of modes, the "out-modes", which become plane waves in the infinite future,

$$
\begin{aligned}
v_{\vec{k}}= & \frac{1}{\sqrt{2 \omega_{\text {out }}}} \exp \left(i \vec{k} \cdot \vec{x}-i \omega_{+} t-i \omega_{-} \delta t \log (2 \cosh t / \delta t)\right) \\
& \times_{2} F_{1}\left(1+i \omega_{-} \delta t, i \omega_{-} \delta t ; 1+i \omega_{\text {out }} \delta t ; \frac{1-\tanh (t / \delta t)}{2}\right) .
\end{aligned}
$$

In terms of these, the field operator is

$$
\phi=\int \frac{d^{d-1} k}{(2 \pi)^{(d-1) / 2}}\left(b_{\vec{k}} v_{\vec{k}}+b_{\vec{k}}^{\dagger} v_{\vec{k}}^{*}\right), \quad \text { where }\left[b_{\vec{k}}, b_{\vec{k}^{\prime}}^{\dagger}\right]=\delta^{d-1}\left(\vec{k}-\vec{k}^{\prime}\right) .
$$

The Bogoliubov transformation that relates these two sets of modes is given by [27]

$$
\begin{aligned}
& u_{\vec{k}}=\alpha_{\vec{k}} v_{\vec{k}}+\beta_{\vec{k}} v_{-\vec{k}}^{\star}, \\
& u_{\vec{k}}^{\star}=\alpha_{\vec{k}}^{\star} v_{\vec{k}}^{\star}+\beta_{\vec{k}}^{\star} v_{-\vec{k}},
\end{aligned}
$$

where

$$
\begin{aligned}
& \alpha_{\vec{k}}=\sqrt{\frac{\omega_{\text {out }}}{\omega_{\text {in }}}} \frac{\Gamma\left(1-i \omega_{\text {in }} \delta t\right) \Gamma\left(-i \omega_{\text {out }} \delta t\right)}{\Gamma\left(-i \omega_{+} \delta t\right) \Gamma\left(1-i \omega_{+} \delta t\right)}, \\
& \beta_{\vec{k}}=\sqrt{\frac{\omega_{\text {out }}}{\omega_{\text {in }}}} \frac{\Gamma\left(1-i \omega_{\text {in }} \delta t\right) \Gamma\left(i \omega_{\text {out }} \delta t\right)}{\Gamma\left(i \omega_{-} \delta t\right) \Gamma\left(1+i \omega_{-} \delta t\right)} .
\end{aligned}
$$

The Heisenberg-picture state of the system is the "in" vacuum,

$$
a_{\vec{k}}|i n, 0\rangle=0 .
$$

We will be interested in analysing several quantities: (i) the two-point correlator of the field at a finite spatial separation (ii) the expectation value of the composite operator $\phi^{2}$ and (iii) the net energy density produced. In fact, the rate of change of the energy density is related to $\left\langle\phi^{2}\right\rangle$ by the Ward identity

$$
\partial_{t}\langle\mathcal{E}\rangle=\frac{1}{2} \partial_{t} m^{2}(t)\left\langle\phi^{2}\right\rangle .
$$

The two point correlation function of the scalar field under the quench reads

$$
\begin{aligned}
&\left\langle i n, 0\left|\phi(\vec{x}, t) \phi\left(\vec{x}^{\prime}, t^{\prime}\right)\right| i n, 0\right\rangle= \int \frac{d^{d-1} k}{(2 \pi)^{d-1}} u_{\vec{k}}(\vec{x}, t) u_{\vec{k}}^{\star}\left(\vec{x}^{\prime}, t^{\prime}\right) \\
&=\int \frac{d^{d-1} k}{(2 \pi)^{d-1}}\left\{\left|\alpha_{\vec{k}}\right|^{2} v_{\vec{k}}(\vec{x}, t) v_{\vec{k}}^{\star}\left(\vec{x}^{\prime}, t^{\prime}\right)+\alpha_{\vec{k}} \beta_{\vec{k}}^{\star} v_{\vec{k}}(\vec{x}, t) v_{-\vec{k}}\left(\vec{x}^{\prime}, t^{\prime}\right)\right. \\
&\left.\quad+\alpha_{\vec{k}}^{\star} \beta_{\vec{k}} v_{-\vec{k}}^{\star}(\vec{x}, t) v_{\vec{k}}^{\star}\left(\vec{x}^{\prime}, t^{\prime}\right)+\left|\beta_{\vec{k}}\right|^{2} v_{-\vec{k}}^{\star}(\vec{x}, t) v_{-\vec{k}}\left(\vec{x}^{\prime}, t^{\prime}\right)\right\} .
\end{aligned}
$$


We will be interested in the relationship of the results of a smooth quench as in (2.2) to that of an instantaneous quench from a mass $m$ to zero mass,

$$
m_{\text {instant }}(t)=m \theta(-t) .
$$

The "in" and "out" modes for such an instantaneous quench have a trivial plane wave profile for before and after the quench, respectively

$$
\begin{aligned}
& u_{\vec{k}}^{\text {instant }}=\frac{1}{\sqrt{2 \omega_{\text {in }}}} e^{i\left(\vec{k} \cdot \vec{x}-\omega_{\text {in }} t\right)}, t \leq 0, \\
& v_{\vec{k}}^{\text {instant }}=\frac{1}{\sqrt{2 \omega_{\text {out }}}} e^{i\left(\vec{k} \cdot \vec{x}-\omega_{\text {out }} t\right)}, t \geq 0 .
\end{aligned}
$$

The Bogoliubov coefficients for the instantaneous quench are determined by demanding that the mode functions and their first derivatives are continuous at $t=0$. This yields:

$$
\alpha_{\vec{k}}^{\text {instant }}=\frac{\omega_{+}}{\sqrt{\omega_{\text {in }} \omega_{\text {out }}}} \quad \text { and } \quad \beta_{\vec{k}}^{\text {instant }}=\frac{\omega_{-}}{\sqrt{\omega_{\text {in }} \omega_{\text {out }}}} .
$$

The correlator for an instantaneous quench can be easily computed using (2.16) (or by directly matching the operator solutions across $t=0$ as in [12]),

$$
\begin{aligned}
\left\langle i n, 0\left|\phi(\vec{x}, t) \phi\left(\vec{x}^{\prime}, t^{\prime}\right)\right| i n, 0\right\rangle & \rightarrow \int \frac{d^{d-1} k}{(2 \pi)^{d-1}} e^{i \vec{k} \cdot\left(\vec{x}-\vec{x}^{\prime}\right)} \\
& \times\left[\frac{e^{-i \omega_{\text {out }}\left(t-t^{\prime}\right)}}{2 \omega_{\text {out }}}+\frac{\left(\omega_{\text {out }}-\omega_{\text {in }}\right)^{2}}{4 \omega_{\text {out }}^{2} \omega_{\text {in }}} \cos \omega_{\text {out }}\left(t-t^{\prime}\right)+\frac{\left(\omega_{\text {out }}^{2}-\omega_{\text {in }}^{2}\right)}{4 \omega_{\text {out }}^{2} \omega_{\text {in }}} \cos \omega_{\text {out }}\left(t+t^{\prime}\right)\right] .
\end{aligned}
$$

The comparison of the instantaneous quench with the smooth fast quench is only meaningful at late times when the variation of the mass in the smooth quench is over, i.e., when $t \gg \delta t$ and $t^{\prime} \gg \delta t$. For such times, the mode functions $v_{\vec{k}}(\vec{x}, t) \rightarrow v_{\vec{k}}^{\text {instant }}$ which are exactly the mode functions for $t>0$ in instantaneous quench.

In what follows, it is useful to look at the behavior of the Bogoliubov coefficients in various regimes.

1. First consider the limit

$$
\omega_{\text {in }} \delta t, \omega_{\text {out }} \delta t \ll 1 .
$$

It may be easily checked that in this limit, the smooth quench Bogoliubov coefficients (2.10) reduce to the instantaneous quench coefficients (2.16), regardless of the value of $|\vec{k}| / m$. This means that the smooth quench approaches an instantaneous quench when $\delta t$ is small compared to all other length scales in the problem. In particular, this means that the momentum space correlators at some momentum $\vec{k}$ will approach the instantaneous correlator only when $m \delta t \ll 1$ as well as $|\vec{k}| \delta t \ll 1$. In [1] we discussed the implications of this for expectation values of local quantities like $\left\langle\phi^{2}(x, t)\right\rangle$ or the energy density. Generically, the small $m \delta t \ll 1$ limit of these local quantities will not agree with the instantaneous quench result since these quantities involve an integration over momenta all way upto the cutoff, and the physical smooth 
quenches, in which we are interested, are fast compared to physical mass scales but slow compared to the cutoff scale. It can be the case, however, that the integrand is a rapidly decaying function. If so, even if we have to integrate to arbitrarily high momenta, the main contributions will come from low momenta and then there would be agreement between the two protocols. For example, as shown in [2], this is what happens in evaluating $\left\langle\phi^{2}\right\rangle$ in $d=3$. In section 5 , we will go back to this discussion and show that in higher dimensions this is not generally true.

2. Now consider the limit

$$
m \delta t \ll 1, \quad|\vec{k}| / m \gg 1, \quad|\vec{k}| \delta t=\text { arbitrary } .
$$

Once again in this limit, the Bogololiubov coefficients (2.10) approach the instantaneous quench coefficients (2.16), for any finite value of $|\vec{k}| \delta t$. In fact, in this limit the coefficients (2.10) behave as

$$
\begin{aligned}
\alpha_{\vec{k}} & \rightarrow 1-\frac{m^{2}}{4 k^{2}}[1-i k \delta t \psi(1-i k \delta t)+i k \delta t \psi(-i k \delta t)]+O\left(m^{4} / k^{4}\right), \\
\beta_{\vec{k}} & \rightarrow \frac{m^{2}}{i k^{2}} k \delta t \Gamma(1-i k \delta t) \Gamma(i k \delta t)+O\left(m^{4} / k^{4}\right),
\end{aligned}
$$

where $\psi(x)$ denotes the digamma function, i.e., $\psi(x)=\partial_{x} \log \Gamma(x)$. For the instantaneous quench, instead, they behave as

$$
\begin{aligned}
\alpha_{\vec{k}}^{\text {instant }} & \rightarrow 1+O\left(m^{4} / k^{4}\right), \\
\beta_{\vec{k}}^{\text {instant }} & \rightarrow-\frac{m^{2}}{4 k^{2}} .
\end{aligned}
$$

Thus to leading order in $m^{2} / k^{2}$, we have $\alpha_{\vec{k}}=1, \beta_{\vec{k}}=0$ for both smooth and instantaneous quenches regardless of the value of $k \delta t$. This is a reflection of the fact that very high momentum modes are not excited by the quench, i.e., to leading order the quench is immaterial for these modes. The subleading terms in (2.20) are of course dependent on $\delta t$. In fact, for finite $k \delta t$, the subleading term in $\alpha_{\vec{k}}$ is $O\left(m^{2} / k^{2}\right)$. However, if in addition we have $k \delta t \ll 1$, this $O\left(\mathrm{~m}^{2} / k^{2}\right)$ term is cancelled, as it should be.

In the next section we discuss the implications of these observations for real space correlation functions.

\section{$3 \quad$ Late time spatial correlators}

In this section, we examine equal time correlation functions at finite spatial separations

$$
C(t, \vec{r}) \equiv\langle\phi(t, \vec{r}) \phi(t, \overrightarrow{0})\rangle
$$

and compare the result for smooth fast quenches and instantaneous quenches at late times. For simplicity, we only explicitly consider the correlators in odd spacetime dimensions in the following. We will consider this correlator in eq. (3.1) in three different situations: the 
first one is the equal time correlator for a smooth quench from a mass $m$ to zero mass, as in eq. (2.2),

$$
C(t, \vec{r})_{\text {smooth }}=\frac{1}{2(2 \pi)^{d-1}} \int d^{d-1} k \frac{e^{i \vec{k} \cdot \vec{r}}}{k}\left\{\left|\alpha_{\vec{k}}\right|^{2}+\left|\beta_{\vec{k}}\right|^{2}+\alpha_{\vec{k}} \beta_{\vec{k}}^{\star} e^{2 i k t}+\alpha_{\vec{k}}^{\star} \beta_{\vec{k}} e^{-2 i k t}\right\},
$$

where $\alpha_{\vec{k}}$ and $\beta_{\vec{k}}$ are given by eq. (2.10). The second quantity is the equal time correlator for an instantaneous quench which can be read off from eq. (2.17),

$$
C(t, \vec{r})_{\text {instant }}=\frac{1}{2(2 \pi)^{d-1}} \int d^{d-1} k e^{i \vec{k} \cdot \vec{r}}\left(\frac{1}{k^{2} \sqrt{k^{2}+m^{2}}}\left(k^{2}+m^{2} \sin ^{2}(k t)\right)\right) .
$$

This correlator was studied in e.g., [12]. Finally, we consider the correlator for a constant mass $m=0$,

$$
C_{\text {const }}(\vec{r})=\frac{1}{2(2 \pi)^{d-1}} \int d^{d-1} k e^{i \vec{k} \cdot \vec{r}} \frac{1}{|\vec{k}|} .
$$

Constant mass correlators are evaluated in detail in appendix A, including the case of $m=0$ - see eq. (A.8). Performing the angular integrals above we find

$$
\begin{aligned}
C(t, \vec{r})_{\text {smooth }} & =\frac{1}{\sigma_{c}} \int \mathcal{C}_{\text {smooth }}(k, t, r) d k \\
& =\frac{1}{\sigma_{c} r^{\frac{d-3}{2}}} \int d k k^{\frac{d-3}{2}} J_{\frac{d-3}{2}}(k r)\left\{\left|\alpha_{\vec{k}}\right|^{2}+\left|\beta_{\vec{k}}\right|^{2}+\alpha_{\vec{k}} \beta_{\vec{k}}^{\star} e^{2 i k t}+\alpha_{\vec{k}}^{\star} \beta_{\vec{k}} e^{-2 i k t}\right\} \\
C(t, \vec{r})_{\text {instant }} & =\frac{1}{\sigma_{c}} \int \mathcal{C}_{\text {instant }}(k, t, r) d k \\
& =\frac{1}{\sigma_{c} r^{\frac{d-3}{2}}} \int d k k^{\frac{d-3}{2}} J_{\frac{d-3}{2}}(k r) \frac{k^{2}+m^{2} \sin ^{2}(k t)}{k \sqrt{k^{2}+m^{2}}} \\
C_{\text {const }}(\vec{r}) & =\frac{1}{\sigma_{c}} \int \mathcal{C}_{\text {const }}(k, r) d k=\frac{1}{\sigma_{c} r^{\frac{d-3}{2}} \int d k k^{\frac{d-3}{2}} J_{\frac{d-3}{2}}(k r)}
\end{aligned}
$$

where $\sigma_{c}=2^{\frac{d+1}{2}} \pi^{\frac{d-1}{2}}$ and $J_{\frac{d-3}{2}}$ is the Bessel function of order $\frac{d-3}{2}$.

Before proceeding with the detailed calculations, let us present our intuitive expectations for the comparison, as well as the results found below. As in section 2, we are considering quenches which take the mass from some fixed initial value $m$ to zero after the quench. There are several different dimensionful parameters at play in our correlators, i.e., $t, \delta t, r=|\vec{r}|$ and $m$, and so first, we wish to clearly specify how the corresponding scales are ordered in our considerations below. First, for the smooth quenches, we are considering the fast quench limit and hence we have $m \delta t \ll 1$. We are also examining the correlators in late time limit and hence $\delta t \ll t$. While these inequalities do not fix a relation between $m$ and $t$, we will only present results for the case $m t>1$. That is, the following discussion explicitly considers quenches where

$$
\delta t \ll 1 / m<t .
$$

We have verified that the general behaviour is the same in regimes where $m t \lesssim 1$, as long as the inequalities for the fast quench and late time limits are both satisfied. 
Given the ordering in eq. (3.8), we still have one remaining scale unspecified, namely the spatial separation $r=|\vec{r}|$. In the following, we compare the correlator (3.1) for fast smooth quenches and that (3.3) for instantaneous quenches for $r$ in different regimes. The natural intuition is that in the Fourier transform, only momenta satisfying $k \lesssim 1 / r$ will contribute significantly to the correlator. Hence given that we are in the fast quench regime with $m \delta t \ll 1$, if we further choose $r \gg \delta t$, then both of the inequalities in eq. (2.18) should be satisfied for the momenta contributing to the correlators. Hence the analysis in the previous section would indicate that at late times, the integrand in eq. (2.13) for the smooth quenches effectively reduces to that in eq. (2.17) matching the correlator for an instantaneous quench. We will explicitly verify that this expectation is realized by numerically comparing the full expression (2.13) for smooth quenches with the instantaneous quench result (2.17) at late times.

Continuing with the above intuition, differences between the late-time correlators for the two different quench protocols are expected to arise in a regime where the spatial separation is comparable to the quench time, i.e., $r \lesssim \delta t$. In view of eq. (3.8), this means that we would be examining the correlator at short distance scales. However, we also found that for very large $k \gg m$ the leading behavior of the Bogoliubov coefficients are in fact independent of the quench rate - see discussion following eq. (2.19). This immediately implies that the leading singularity at small $r$ is independent of any quench protocol and therefore one gets the same singular behaviour as the constant mass correlators in this regime, namely, $C(t, \vec{r}) \propto 1 / r^{d-2}-$ see appendix A. Hence the leading behaviour in the correlators produced by the smooth and instantaneous quenches again agrees in this regime. As we will show below, the subleading singularities are in fact different in high dimensions.

The interesting regime where the difference between a smooth and instantaneous quench would show up is therefore the intermediate values of $r$. To study this difference it is convenient to eliminate the leading small- $r$ contribution by calculating the difference between the two quench correlators, i.e., $C_{\text {smooth }}(t, \vec{r})-C_{\text {instant }}(t, \vec{r})$, or alternatively by subtracting the fixed mass correlator from each of the quench correlators individually. As we describe in detail below, this difference of the late-time correlators indicates that the subleading behaviour, in fact, also agrees for $d=3$ but a small finite difference arises for $d=5$. For $d=7$ and higher dimensions, the difference still diverges in the limit $r \rightarrow 0$.

\subsection{Numerical results for various dimensions}

We now evaluate the $k$ integrals in eqs. (3.5)-(3.7) numerically. However the integrands are typically oscillating rapidly with a growing envelope. Hence to evaluate the integral properly, we need to regulate the integral. We do so by introducing a convergence factor $\exp (-a k)$ with $a>0$ and evaluating the integral in the limit $a \rightarrow 0$, as discussed in appendix A. Using this regulator, the integral in eq. (3.7) can be evaluated to yield $C_{\text {const }}(\vec{r}) \propto 1 / r^{d-2}$, as in eq. (A.8). This is the standard massless propagator in $d$ dimensions. For our numerical calculations, we typically use $a=10^{-3}$, which we can verify is small enough that the integrals accurately converge to their limiting values.

As noted in the above discussion, for large $k \gg m, \mathcal{C}_{\text {smooth }}(k)$ and $\mathcal{C}_{\text {instant }}(k)$ are essentially identical to $\mathcal{C}_{\text {const }}(k)$, implying that the leading divergence in all the corresponding 


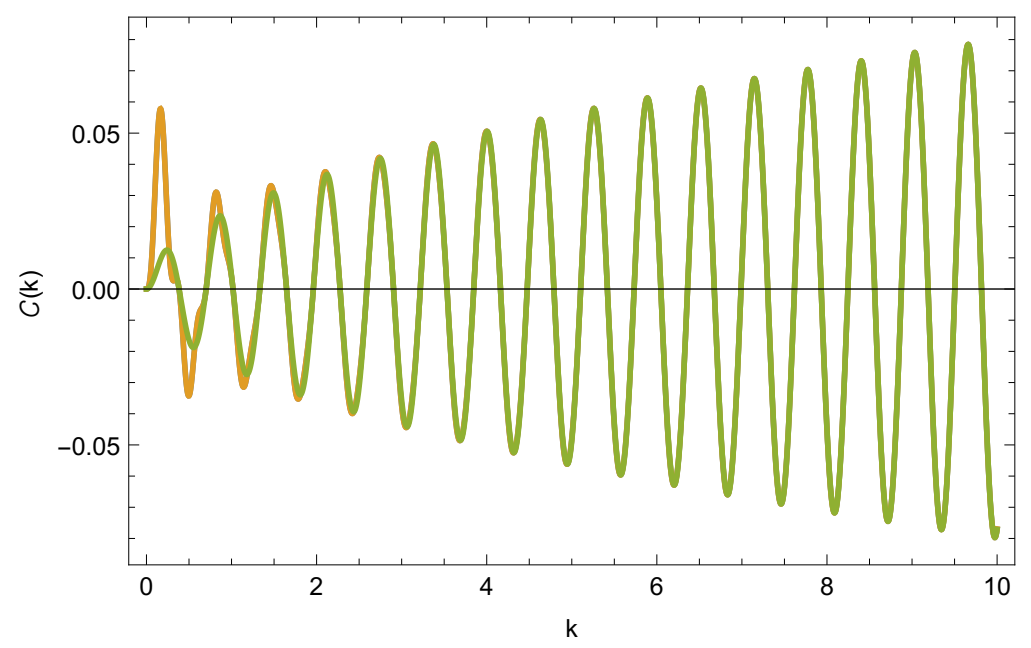

Figure 1. (Colour online) Integrands $\mathcal{C}_{\text {instant }}$ (blue), $\mathcal{C}_{\text {smooth }}$ (yellow) and $\mathcal{C}_{\text {const }}$ (green) of the instantaneous quench, smooth quench and constant massless case, for $d=5, t=10, r=10$ and $\delta t=1 / 20$ as a function of momentum $k$ - all dimensionful quantities are given in units of the initial mass $m$. All three integrands are nearly identical for large momenta. Further the instantaneous and smooth quench curves overlap at all scales.

correlators is $1 / r^{d-2}$ for small $r$. Hence, the integrands are very close to each other for a large range of $k$, as illustrated in figure 1 .

Therefore, in order to highlight the differences between the smooth and instantaneous quenches, we will subtract off eq. (3.7) to define for both cases

$$
\tilde{C}(t, r)=\frac{1}{\sigma_{c}} \int \tilde{\mathcal{C}}(k, t, r) d k \equiv C(t, r)-C_{\text {const }}(r)=\frac{1}{\sigma_{c}} \int\left(\mathcal{C}(k, t, r)-\mathcal{C}_{\text {const }}(k, t, r)\right) d k .
$$

In terms of the integrands, subtracting $\mathcal{C}_{\text {const }}(k)$ suppresses the growing oscillations at large $k$ that, e.g., we see in figure 1 . In the full correlator, this subtraction removes the divergent behaviour at $r \rightarrow 0$, which makes it easier to identify differences in the finite remainder. If this behaviour was not removed, for instance, both the instantaneous and the smooth quench would both grow as $1 / r^{d-2}$ in exactly the same way as $r \rightarrow 0$ and it would be extremely difficult to find any differences between the correlators for the two different quench protocols in this regime. ${ }^{3}$ In comparing the subtracted integrands $\tilde{\mathcal{C}}$ below, we start by considering $d=5$.

In figure 1 , as well as the good agreement between the quench correlators and the constant massless correlator at large $k$, we see that the two integrands, $\mathcal{C}_{\text {smooth }}(k)$ and $\mathcal{C}_{\text {instant }}(k)$, agree for throughout the momentum range shown there. Given our discussion at the opening of this section, we only expect differences to arise when $k \gtrsim 1 / \delta t$. One way to illustrate these differences is to make $\delta t$ larger, as illustrated in figure 2. Panel (a) shows the subtracted integrands in the range $0 \leq k \leq 2$ with the same parameters

\footnotetext{
${ }^{3}$ This would be analogous to analysing the bare expectation value $\left\langle\phi^{2}\right\rangle$ instead of the renormalized quantity $\left\langle\phi^{2}\right\rangle_{\text {ren }}$ in $[1,2]$. Of course, the interesting physical quantity is in the renormalized expectation value - see further discussion in section 3.2.
} 


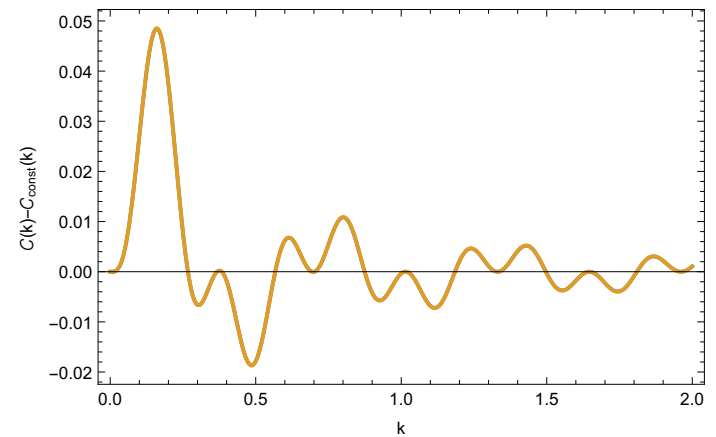

(a) $\delta t=1 / 20$.

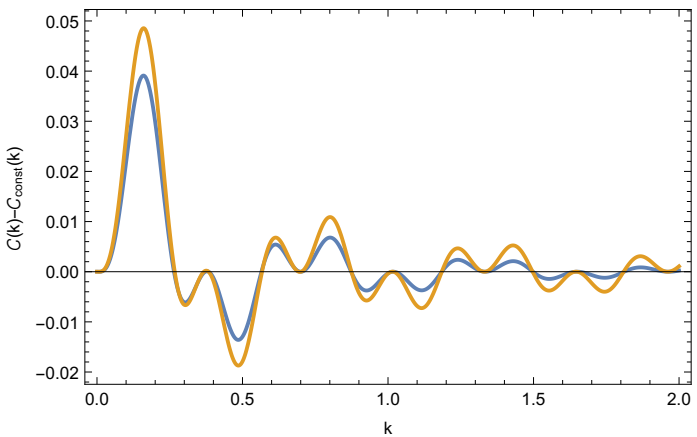

(b) $\delta t=1 / 2$.

Figure 2. (Colour online) Subtracted integrands as a function of (small) momentum $k$. In this case we are plotting for $d=5, t=10, r=10$ (with the units set by $m$ ). The yellow line corresponds to the instantaneous quench while the blue one to the smooth. Panel (a) shows that no detectable differences appear with $\delta t=1 / 20$ in the range $0 \leq k \leq 2$. However, in panel (b), minor differences occur in this range when $\delta t=1 / 2$.

as used in figure 1, i.e., $m t=10, m r=10$ and $m \delta t=1 / 20$ for $d=5$, and the two curves precisely overlap in this momentum range. The only change of parameters in panel (b) is that here $m \delta t=1 / 2$ and we clearly see that small differences appear between the integrand for the smooth quench and that for the instantaneous quench. Note, however, that with $m \delta t=1 / 2$, the smooth quench only barely satisfies eq. (2.18) and so while useful to illustrate possible differences, this example is not really in the fast quench regime.

Focusing on the parameters $m t=10, m r=10$ and $m \delta t=1 / 20$ (for $d=5$ ), we see in figure 3 that the subtracted integrands continue to agree for much larger values of $k$. However, with $k / m \sim 20$, differences are clearly visible in panel (b). However, comparing the vertical scale in these two plots to that in panel (a) of figure 1, we see that these large $k$ contributions to the subtracted integrand are highly suppressed. ${ }^{4}$ Hence the differences should not be expected to contribute to the full integral, i.e., they should not produce significant differences in the position-space correlators.

It turns out that examining the subtracted integrands for $d=3$ yields essentially the same results. On the other hand, the situation is also similar in $d=7$ for long distances. As we decrease $r$, for $d=7$, we see analogous effects to those in figure 4, i.e., the two integrands become clearly different. However, as we will see, after integration, the behaviour of the correlator at small distances is very different depending on the dimension.

Given that we understand the behaviour of the (subtracted) integrands, let us now compare the (position-space) correlators for instantaneous and smooth quenches at different values of $r$, as shown in figure 5. In the figure, we have chosen $m t=10$ and $m \delta t=1 / 20$ while $m r$ varies from 10 to 0.001 . As we have expected, the figure shows that the difference between the correlators goes to zero, or at least is of order $O\left(\delta t^{2}\right)$, at large $m r$. The results for $d=3$ indicate that the difference remains vanishingly small for all values of $r$. In the case

\footnotetext{
${ }^{4}$ Further, these large $k$ contributions are also highly oscillatory, so they will tend to cancel out in the integral.
} 


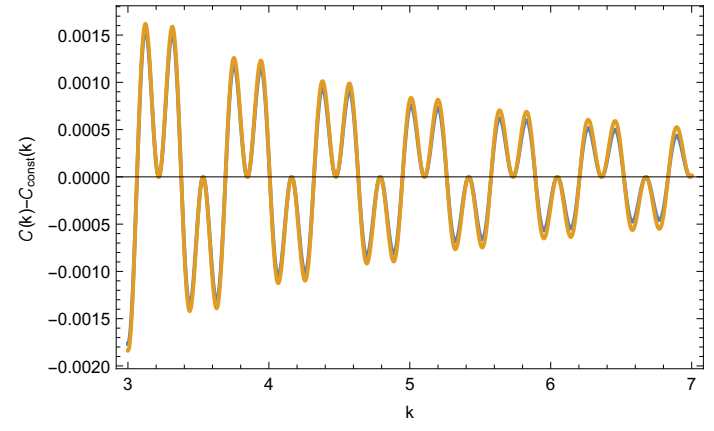

(a) Intermediate momentum.

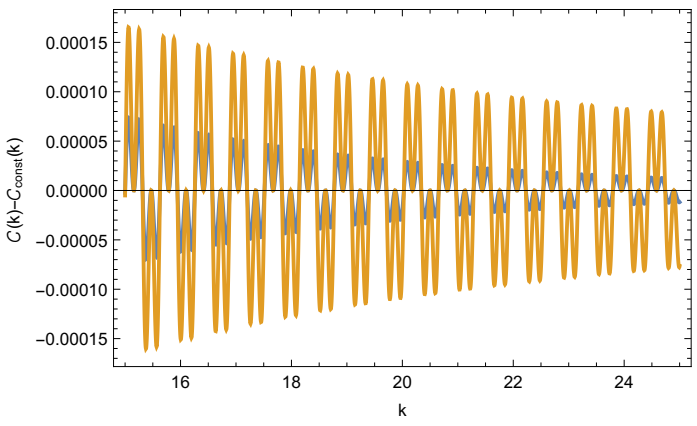

(b) Large momentum.

Figure 3. (Colour online) Subtracted integrands as a function of momentum $k$. In this case we are plotting for $d=5, t=10, r=10$ and $\delta t=1 / 20$ (with the units set by $m$ ). The blue line corresponds to the smooth quench while the yellow one to the instantaneous quench. Panel (a) shows an intermediate regime where no significant differences between the two integrands are apparent. Visible differences appear for larger $k \gtrsim 1 / \delta t$, in panel (b).

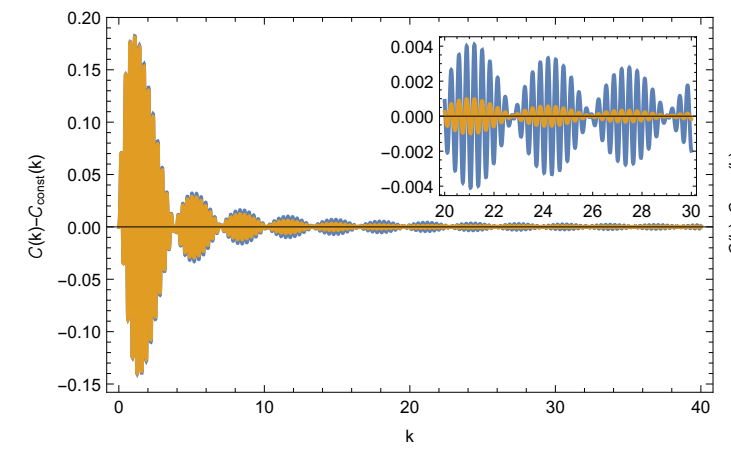

(a) $m r=1$.

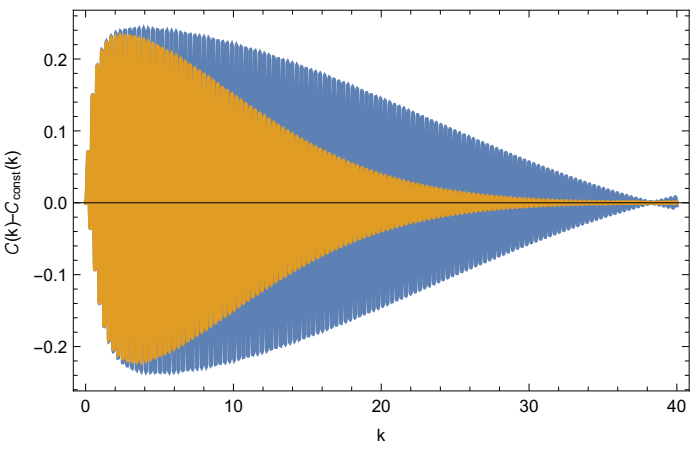

(b) $m r=0.1$.

Figure 4. (Colour online) Regulated integrands as a function of momentum $k$ for $d=5$ and small separation. The blue line corresponds to the smooth quench while the yellow one to the instantaneous. At small distances the two integrands are clearly different from each other, even at scales $k \delta t \leq 1$.

of $d=5$, a small but finite difference appears to develop as $r$ goes to zero. The differences in figure 5 are most evident for $d=7$. In this case, the relative difference is already of order one at $m r=0.1$ and the trend in the figure is that it continues to grow at smaller $r$. Our expectation is that in fact, this difference will in fact diverge as $r \rightarrow 0$. This conclusion comes from comparing to the late-time behaviour of expectation value $\left\langle\phi^{2}\right\rangle_{\text {ren }}$ in the next section.

In summary then, we have explicitly shown that at times long after the quench, the correlators generated by instantaneous and the smooth fast quenches are identical at large separations. As might be expected, differences only appear at separations of the order the quench time $\delta t$. Further these differences are small in lower dimensions, e.g., $d=3,5$, but can be quite dramatic in higher dimensions. Interestingly, for $d=3$ and 5 , the subtracted correlator of both the instantaneous and the smooth quench (with the $m r=0$ piece 


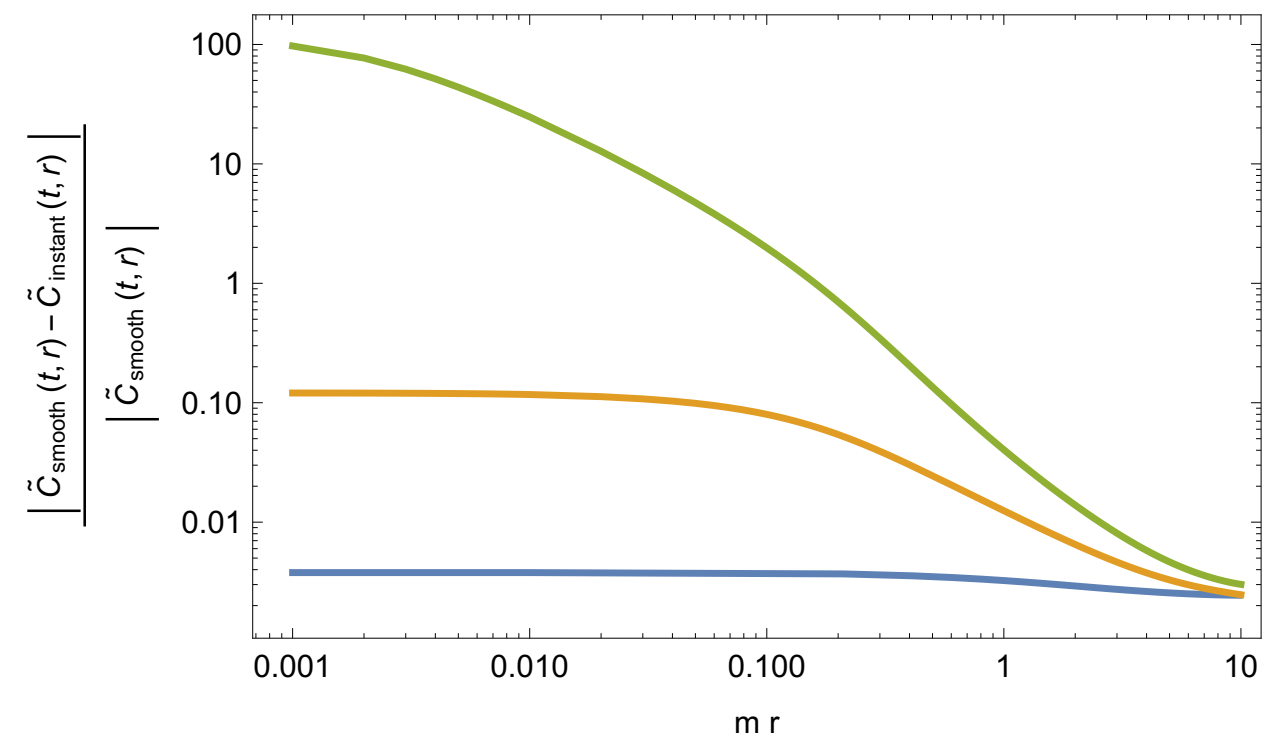

Figure 5. (Colour online) Difference between the late-time correlators for smooth and instantaneous quenches as a function of the separation distance $r$. The blue line corresponds to the $d=3$ case while the yellow one belongs to $d=5$ and $d=7$ is shown in green. We are using $m t=10$ with $m \delta t=1 / 20$. In $d=3$ and $d=5$, the difference remains small for any value of $r$, while in $d=7$, it seems to diverge as $r \rightarrow 0$.

subtracted out) agree to a rather high precision for any distance $m r$. As we increase $m r$ both answers become even closer.

\subsection{Small $r$ behavior and counterterms for local operators}

In $[1,2]$, we studied the UV divergences appearing in $\left\langle\phi^{2}\right\rangle$ with a momentum cut-off in great detail. Intuitively, one can think that the correlator at small $r$ provides a point-splitting regulator of the same quantity. Hence, the divergences in the correlator at small $r$ should be related to the UV divergences of the local quantity $\left\langle\phi^{2}\right\rangle$. In this subsection, we make this connection precise.

Let's start with the constant massless correlator, (3.7). For small $r$ we can expand the Bessel function to get,

$$
J_{\frac{d-3}{2}}(k r)=(k r)^{\frac{d-3}{2}}\left(\frac{2^{\frac{3-d}{2}}}{\Gamma\left(\frac{d-1}{2}\right)}+O\left((k r)^{2}\right)\right) .
$$

Inserting this in eq. (3.7), we get an integral of $k^{d-3}$, exactly the same as the leading divergence in $\left\langle\phi^{2}\right\rangle$.

Let us first recall the set of counterterms that we found in $[1,2]$ for $\left\langle\phi^{2}\right\rangle$. There the leading divergences in momentum space were determined by performing an adiabatic expansion in time derivatives and then expanding our results for large momentum. We then found that this was the same as expanding the integrand of $\left\langle\phi^{2}\right\rangle$. So, to be more specific, in the "in"-basis, the integrand took the form $\left.\left.k^{d-2} \omega_{\text {in }}^{-1}\right|_{2} F_{1}\right|^{2}$ and for large $k$ we 
found [2]

$$
\begin{aligned}
\left.\left.\frac{k^{d-2}}{\omega_{\text {in }}}\right|_{2} F_{1}\right|^{2} \simeq & k^{d-3}-\frac{k^{d-5}}{2} m^{2}(t)+\frac{k^{d-7}}{8}\left(3 m^{4}(t)+\partial_{t}^{2} m^{2}(t)\right) \\
& -\frac{k^{d-9}}{32}\left(10 m^{6}(t)+\partial_{t}^{4} m^{2}(t)+10 m^{2}(t) \partial_{t}^{2} m^{2}(t)+5 \partial_{t} m^{2}(t) \partial_{t} m^{2}(t)\right)+\cdots
\end{aligned}
$$

Above, we are giving all the terms needed to regulate the expectation value up to $d=9$. Apart from the divergent terms in the constant mass expectation value (that will appear as zeroth order in the adiabatic expansion), they include terms with time derivatives of the mass profile which produce divergences in $\left\langle\phi^{2}\right\rangle$ for $d \geq 6$. These (perhaps surprising) terms were carefully analysed in [2]. Now we can express the bare expectation value of the local operator in terms of an energy cut-off $\Lambda$, obtained by integrating the momentum integral up to a maximum value $k_{\max } \simeq \Lambda$. This yields

$$
\begin{aligned}
\left\langle\phi^{2}\right\rangle \simeq & \frac{1}{2^{d-1} \pi^{\frac{d-1}{2}} \Gamma\left(\frac{d-1}{2}\right)}\left(\frac{\Lambda^{d-2}}{d-2}-\frac{\Lambda^{d-4}}{d-4} \frac{m^{2}(t)}{2}+\frac{\Lambda^{d-6}}{d-6} \frac{3 m^{4}(t)+\partial_{t}^{2} m^{2}(t)}{8}-\right. \\
& \left.-\frac{\Lambda^{d-8}}{d-8} \frac{10 m^{6}(t)+\partial_{t}^{4} m^{2}(t)+10 m^{2}(t) \partial_{t}^{2} m^{2}(t)+5 \partial_{t} m^{2}(t) \partial_{t} m^{2}(t)}{32}+\cdots\right) .
\end{aligned}
$$

Working in terms of the "in" modes, the smooth quench correlator can be expressed as

$$
\begin{aligned}
C(t, \vec{r})_{\text {smooth }} & =\frac{1}{\sigma_{c} r^{\frac{d-3}{2}}} \int d k \frac{k^{(d-1) / 2}}{\omega_{\text {in }}} J_{\frac{d-3}{2}}(k r)\left|{ }_{2} F_{1}\right|^{2}, \\
& =\frac{1}{\sigma_{c} r^{\frac{d-3}{2}}} \int d k \frac{1}{k^{\frac{d-3}{2}}} J_{\frac{d-3}{2}}(k r) \frac{k^{d-2}}{\omega_{\text {in }}}\left|{ }_{2} F_{1}\right|^{2},
\end{aligned}
$$

where in the last line we have presented the integrand in a way which allows us to make use of the above expansion in eq. (3.13). So we have all the ingredients to take the limit from the spatial correlator to the expectation value. In particular, if we take the $r \rightarrow 0$, then the Bessel function can be replaced for its zeroth order expansion shown in eq. (3.10). The powers of $k r$ in eq. (3.10) will cancel those same powers appearing in eq. (3.13), while the numerical factors will turn the $\sigma_{c}$ into a $\sigma_{s} .{ }^{5}$ Then we can use eq. (3.11) to expand for large $k$ and find that the correlator behaves exactly in the same way as $\left\langle\phi^{2}\right\rangle$ when $r \rightarrow 0$. In fact,

$$
\begin{aligned}
C(t, \vec{r}) \underset{r \rightarrow 0}{\longrightarrow} & \frac{1}{\sigma_{s}} \int d k\left(k^{d-3}-\frac{k^{d-5}}{2} m^{2}(t)+\frac{k^{d-7}}{8}\left(3 m^{4}(t)+\partial_{t}^{2} m^{2}(t)\right)\right. \\
& \left.-\frac{k^{d-9}}{32}\left(10 m^{6}(t)+\partial_{t}^{4} m^{2}(t)+10 m^{2}(t) \partial_{t}^{2} m^{2}(t)+5 \partial_{t} m^{2}(t) \partial_{t} m^{2}(t)\right)+\cdots\right) .
\end{aligned}
$$

Of course for any finite (positive) $r$ the correlator will be UV finite. So in principle we should be able to perform the integral over momenta to obtain a series expansion in inverse powers of $r$. We now show that this small- $r$ expansion of the correlator is directly related

\footnotetext{
${ }^{5}$ We remind the reader that $\sigma_{s}$ is a numerical constant that depends on the space-time dimension $d$ and was used in $[1,2]$ to normalize the expectation value of $\phi^{2}$. Explicitly, $\sigma_{s} \equiv \frac{2(2 \pi)^{d-1}}{\Omega_{d-2}}=\frac{\Gamma\left(\frac{d-1}{2}\right)}{2^{d-1} \pi^{\frac{d-1}{2}}}$, where $\Omega_{d-2}$ is the angular volume of a unit $(d-2)$-dimensional sphere.
} 
to the large- $k$ expansion for the expectation value. Let us take expression in eq. (3.13) and replace $\left.\left.k^{d-2} \omega_{\text {in }}^{-1}\right|_{2} F_{1}\right|^{2}$ with the expansion given in eq. (3.11). The integrand is composed of a series of terms which are constants (not functions of momentum) multiplying the Bessel function and some power of $k$. For fixed value of $r$, we can integrate that expression, using

$$
\int_{0}^{\infty} k^{\alpha} J_{\frac{d-3}{2}}(k r) d k=\frac{2^{\alpha}}{r^{\alpha+1}} \frac{\Gamma\left(\frac{1}{4}(d+2 \alpha-1)\right)}{\Gamma\left(\frac{1}{4}(d-2 \alpha-1)\right)} .
$$

Now, as an example, consider the leading divergence, i.e., $k^{d-3}$, which gives

$$
\frac{1}{\sigma_{c} r^{\frac{d-3}{2}}} \int_{0}^{\infty} k^{\frac{d-3}{2}} J_{\frac{d-3}{2}}(k r) d k=\frac{1}{\sigma_{c}} \frac{2^{\frac{d-3}{2}} \Gamma\left(\frac{d}{2}-1\right)}{\sqrt{\pi}} \frac{1}{r^{d-2}},
$$

after using eq. (3.15) with $\alpha=(d-3) / 2$ and some algebra. This shows that UV divergences of the local quantity $\left\langle\phi^{2}\right\rangle$ appear as inverse powers of $r$ in the finite spatial separation correlator, i.e., $r$ plays the role of a point-splitting regulator, replacing the momentum cut-off $\Lambda$ in eq. (3.13). In the above example we showed that the leading divergence is proportional to $r^{d-2}$. We can proceed to do the integral for $\alpha=\frac{d-3}{2}-2$. This would correspond to divergences proportional to $k^{d-5}$ and will lead to a term in the spatial correlator which is inversely proportional to $r^{d-4}$. Note that in general, eq. (3.15) maps the integral over $k^{\alpha}$ to the power $1 / r^{\alpha+1}$. Also note that there is an important difference between the leading divergence and the rest of the divergent terms. All of the subleading divergences are proportional either to the instantaneous mass $m(t)$ or to time derivatives of $m(t)$, while the leading divergence is independent of $m(t)$. This means that the leading term as $r \rightarrow 0$ will be inversely proportional to $r^{d-2}$ but then there will be subleading terms inversely proportional to $r^{d-4}, r^{d-6}$, etc., that will also contain factors of the mass and its derivatives. Explicitly we find

$$
\begin{aligned}
& C(t, r) \simeq \frac{\Gamma\left(\frac{d-4}{2}\right)}{8 \pi^{d / 2}}\left(\frac{d-4}{r^{d-2}}-\frac{1}{r^{d-4}} \frac{m^{2}(t)}{2}+\frac{1}{3(d-6) r^{d-6}} \frac{3 m^{4}(t)+\partial_{t}^{2} m^{2}(t)}{8}-\right. \\
& \left.-\frac{1}{15(d-6)(d-8) r^{d-8}} \frac{10 m^{6}(t)+\partial_{t}^{4} m^{2}(t)+10 m^{2}(t) \partial_{t}^{2} m^{2}(t)+5 \partial_{t} m^{2}(t) \partial_{t} m^{2}(t)}{32}+\cdots\right) .
\end{aligned}
$$

In particular, note that to analyse the structure of the correlator in $d=7$, we would have to take into account a term that is proportional to the second derivative of the mass, that will increase as $1 / r$ when $r \rightarrow 0$. This will be important to understand the behaviour of the correlator in section 4 . Also note that this term is also subleading compared to the term proportional to $m^{2} / r^{3}$, that comes second in the expansion of eq. (3.18).

Finally let us note that, even though the momentum cut-off expression in eq. (3.13) and the $r$ expansion expression in eq. (3.18) are similar in form, there is no simple way to relate the energy scale $\Lambda$ with the point-splitting regulator $1 / r$. Rather, equating these two equations we get,

$$
\Lambda \simeq \frac{\Gamma(d-1)^{\frac{1}{d-2}}}{r}\left(1+\frac{\left(1-\Gamma(d-2) \Gamma(d-1)^{-\frac{d-4}{d-2}}\right)}{2(d-4) \Gamma(d-1)^{\frac{2}{d-2}}} m^{2}(t) r^{2}+\cdots\right) .
$$

This simply points-out that these divergent terms are unphysical and that these two regularization schemes have slightly different counterterms. 


\section{Universal scaling in quenched spatial correlators}

In $[1,2]$ we found an interesting set of universal scaling relations for the expectation value of the quenched operator $\left\langle\phi^{2}\right\rangle$ and the energy density. For the quenches considered in this work, this scaling takes the form $\left\langle\phi^{2}\right\rangle_{\text {ren }} \sim m^{2} \delta t^{4-d}$. We also found analytic leading order expressions for this expectation value in the case of $\delta t \rightarrow 0$. For odd spacetime dimensions, we found

$$
\left\langle\phi^{2}\right\rangle_{\mathrm{ren}}=(-1)^{\frac{d-1}{2}} \frac{\pi}{2^{d-2}} \partial_{t}^{d-4} m^{2}(t)+O\left(\delta t^{6-d}\right),
$$

which reproduces the above scaling with the mass profile 2.2 we are considering. On the other hand, we have already shown how the spatial correlator approaches the expectation value of $\phi^{2}$ as the separation distance goes to zero. Then an interesting question to ask is whether there are any signs of the universal scaling in the spatial correlator.

To investigate on this question here, we will concentrate on early times, since this is the regime where scaling of the local quantities hold. Now we need a suitable object to compute. We remind the reader that the scalings hold for renormalized expectation values. Given that the bare expectation values are UV divergent, we had to add suitable counterterms to eliminate those divergences. On the contrary the spatial correlator is finite for any finite separation $r$. However, as discussed in the previous section, the counterterms are in precise correspondence with the small $r$ expansion of the correlator. In particular the leading UV divergence of $\left\langle\phi^{2}\right\rangle$ goes as $\Lambda^{d-2}$, which reflects the leading small $r$ divergence of the correlator behaving as $1 / r^{d-2}$. So from this perspective, the scaling behaviour is only exhibited in a higher order term, which remains finite as $r \rightarrow 0$. However, we may still see the scaling in the correlator by subtracting a suitable fixed mass correlator to remove the terms which diverge in the small $r$ limit. It turns out that in order to eliminate these divergences (which are proportional to powers of $m^{2}$ ) the interesting object to compute is the difference of the spatial quenched correlator at time $t$ with the correlator at a constant value of the mass equal to the instantaneous mass at that time $t$. The latter fixed mass correlator has been computed in appendix A and one finds,

$$
C_{\text {fixed }}(\vec{r})=\frac{1}{\sigma_{c} r^{\frac{d-3}{2}}} \int \frac{k^{\frac{d-1}{2}} d k}{\sqrt{k^{2}+m^{2}(t)}} J_{\frac{d-3}{2}}(k r) .
$$

In order to numerically integrate the correlator, we will go back to the "in" basis - see eq. (3.13) - to obtain

$$
C(t, \vec{r})=\frac{1}{\sigma_{c} r^{\frac{d-3}{2}}} \int d k \frac{k^{(d-1) / 2}}{\sqrt{k^{2}+m^{2}}} J_{\frac{d-3}{2}}(k r)\left|{ }_{2} F_{1}\right|^{2} .
$$

First consider the correlator at $t=0$. In figure 6 , we computed $C(t=0, r)-C_{\text {fixed }}(r)$ for a wide range of values of $r$ and $\delta t$ for $d=5$ and $d=7$. We see a very interesting behaviour. Basically we can divide the correlator in three different regions: (i) $r>\delta t$, (ii) $r<\delta t<m^{-1}$ and (iii) $r<\delta t \sim m^{-1}$.

For $\delta t<r$ we see that the correlator is essentially a constant independent of $\delta t$ that depends on $r$. In fact it turns out that this constant value is exactly the same value of the 
instantaneous quench correlator evaluated at $t=0$. Recall that the instantaneous quench correlator at $t=0$ is exactly the same as the constant mass correlator with $m^{2}=m_{\mathrm{in}}^{2}$. This coincidence might lead the reader to think that this behaviour is something special for $t=0$. However, in what follows, we will show that is the general behaviour of the correlator at any finite $t / \delta t$, as long as $\delta t \ll r \ll 1 / m$.

Let's start by fixing the dimensionless time $\tau=t / \delta t$. Now we want to analyse the $r$ and $\delta t$ dependence of the following object:

$$
C(t, \vec{r})-C_{\text {fixed }}(\vec{r})=\frac{1}{\sigma_{c} r^{\frac{d-3}{2}}} \int d k k^{\frac{d-1}{2}} J_{\frac{d-3}{2}}(k r)\left(\frac{1}{\sqrt{k^{2}+m_{\text {in }}^{2}}}\left|{ }_{2} F_{1}\right|^{2}-\frac{1}{\sqrt{k^{2}+m^{2}(\tau)}}\right) .
$$

Note that the first term inside the big brackets has $m_{\text {in }}^{2}$ in the denominator while the fixed mass part carries an $\mathrm{m}^{2}$ equal to that at the particular time we are considering.

The second important thing to notice is where is the time-dependence in the quenched correlator. The only place where $\tau$ appears explicitly is in the last argument of the hypergeometric function. Recall that

$$
{ }_{2} F_{1} \equiv{ }_{2} F_{1}\left(1+i \omega_{-} \delta t, i \omega_{-} \delta t ; 1-i \omega_{\text {in }} \delta t ; \frac{1+\tanh (t / \delta t)}{2}\right) .
$$

Then, if we fix $\tau$ by inserting any finite number (or zero) in that last argument, we are left with an object that depends only on $\delta t$ and $r$, and we can take the desired limit without any problem. So consider now $\delta t \ll r$. We get that limit by considering the limit of the hypergeometric function with $\delta t \rightarrow 0$. As the second argument is proportional to $\delta t$, to lowest order all the terms in the infinite sum will vanish but the first one, so we just get that when $\delta t \rightarrow 0,{ }_{2} F_{1}=1+O(\delta t)$. Note that this argument is valid only in the case where we fix $\tau$ and effectively there is no $\delta t$ dependence in the last argument. Then, after taking this first limit, we are left with

$$
C(t, \vec{r})-C_{\text {fixed }}(\vec{r}) \approx \frac{1}{\sigma_{c} r^{\frac{d-3}{2}}} \int d k k^{\frac{d-1}{2}} J_{\frac{d-3}{2}}(k r)\left(\frac{1}{\sqrt{k^{2}+m_{\text {in }}^{2}}}-\frac{1}{\sqrt{k^{2}+m^{2}(\tau)}}\right) .
$$

But this is nothing more than $C_{\text {fixed }}\left(m^{2}=m_{\text {in }}^{2}\right)-C_{\text {fixed }}\left(m^{2}=m^{2}(\tau)\right)$ ! So, at any time we get that for $\delta t \ll r$ our object becomes the difference of two fixed mass correlators. In particular, it becomes independent of $\delta t$ and that explains the horizontal dashed lines of figure 6 .

However we can even go further and consider the limit of $r \ll 1 / m$. As it is just the limit for fixed mass correlators we can extract it directly from eq. (A.7) in appendix A. As we are subtracting two fixed mass correlators, the leading term in the expansion, i.e., the one proportional to $r^{d-2}$, will cancel and the leading contribution will come from the first subleading term:

$$
C(t, \vec{r})-C_{\text {fixed }}(\vec{r}) \approx \frac{1}{\sigma_{c}}\left(\frac{m_{\text {in }}^{2}}{2 r^{d-4}}-\frac{m^{2}(\tau)}{2 r^{d-4}}\right)
$$


In our plots of figure 6 , we have $m_{\text {in }}=1$ and $m(\tau=0)=1 / 2$, so the difference of correlators in the limit of $\delta t \ll r \ll 1 / m$ should go as $\left(4 \sigma_{c} r^{d-4}\right)^{-1}$, which agrees with the values that the horizontal lines take in the plots. We can add that the agreement gets more exact as $r$ takes smaller and smaller values.

Note, however, that eq. (4.6) is valid for any value of $m$, as long as $r / \delta t \gg 1$, but eq. (4.7) also needs $r m \ll 1$. If we concentrate in a regime where $r m \sim 1$ (see bottom line in each plot of figure 6), then we should expect eq. (4.6) to hold rather than eq. (4.7). In fact, for $r=m=1$, the bottom dashed line in figure 6 corresponds to approximately 0.10 and 0.20 for $d=5$ and $d=7$, respectively. This is in perfect agreement with eq. (4.6) and clearly differs from the 0.25 that eq. (4.7) is predicting.

To further support our claim we provide equivalent plots but at different times for $d=5$. In figure 7 , we examine the results for $\tau=1 / 2$ and $\tau=1 / 4$. In these cases, the expectation is that the limiting value would be

$$
C(t, \vec{r})-C_{\text {fixed }}(\vec{r}) \approx \frac{m_{\text {in }}^{2}-m^{2}(\tau)}{\sigma_{c} r}=\frac{1-(1 / 2-1 / 2 \tanh \tau)}{\sigma_{c} r},
$$

and that's what arises in this figure. ${ }^{6}$

The second regime $r<\delta t<1 / m$ leads to the most interesting behaviour. The correlator now exhibits exactly the same scaling as the $\phi^{2}$ expectation value. The solid lines we see in the plots are exactly the lines that come from evaluating eq. (4.1) for $d=5$ and $d=7$ with the present mass quench profile - see eq. (2.2). This means that in this regime exactly the same universal scaling we've been discussing for $\phi^{2}$ is reproduced in the spatial correlator.

Finally, at least for $d=5$, we see that when $\delta t \sim 1 / m$ this behaviour breaks down and our calculation goes away from the universal scaling line. It would be interesting to understand better this slow regime as it could be connected to other set of important universal scalings in quantum quenches: namely, the Kibble-Zurek scaling [7, 8] and this would give a connection between the fast and the slow regime universality in quantum quenches. We hope to report on this in the near future.

\section{$5 \quad$ Late time behaviour of $\phi^{2}$}

In [2], we found some interesting behaviour for the expectation value of $\phi^{2}$ when we examined $d=4$ at late times. Essentially, the expectation value for the smooth quench did not depend on the quench duration $\delta t$. This result led us to conjecture that this late time behaviour agrees with that in an instantaneous quench.

In this section, we return to this issue, first by reviewing the $d=3$ result and then by considering late time behaviour for higher dimensions. We will show that the agreement between smooth and instantaneous quenches found in $d=3$ does not generally occur in higher dimensions.

\subsection{Review of $d=3$}

The starting point will be to consider the correlator in eq. (2.17) for instantaneous quench, and evaluate this expression at coincident points in space and time, i.e., $\vec{x}=\vec{x}^{\prime}, t=t^{\prime}$.

\footnotetext{
${ }^{6}$ For $\tau=1 / 2$, then $C(t, \vec{r})-C_{\text {fixed }}(\vec{r}) \approx 0.365529 /\left(\sigma_{c} r\right)$ and for $\tau=1 / 4$, the value is $0.31123 /\left(\sigma_{c} r\right)$.
} 


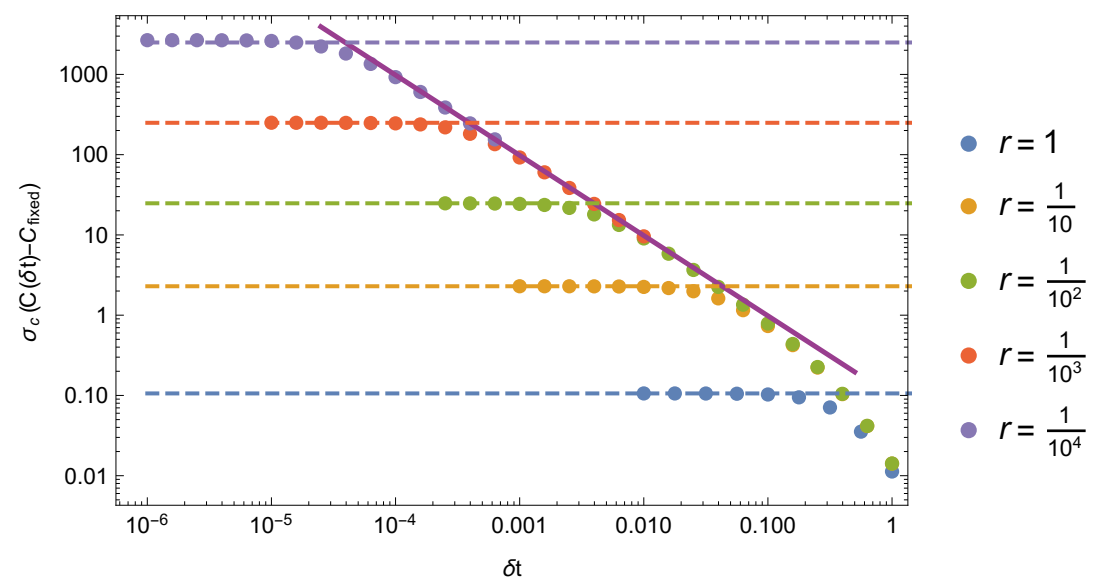

(a) $d=5$.

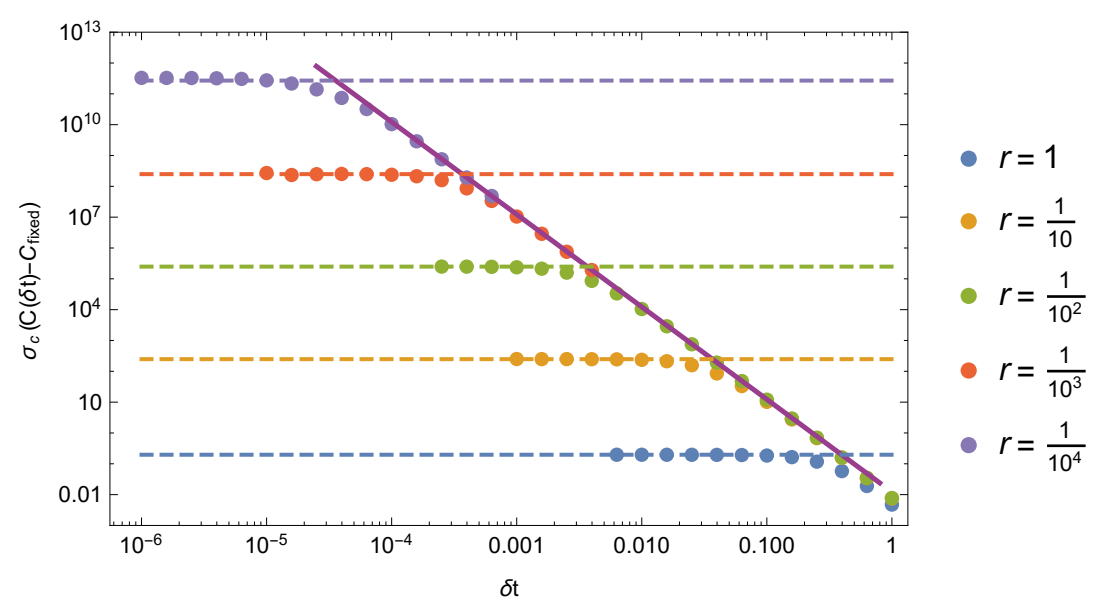

(b) $d=7$.

Figure 6. (Colour online) Spatial correlator under a smooth quench at $t=0$ as a function of both $\delta t$ and the distance separation $r$. In each case, we are subtracting the fixed mass correlator with $m^{2}=$ $1 / 2$. The dashed lines correspond to computing the instantaneous quench correlator at $t=0$ for the different separations $r$, that is the same as computing the fixed mass correlator with $m=m_{\text {in }}=1$. The purple solid line shows the analytic leading order contribution to $\left\langle\phi^{2}\right\rangle$, given by eq. (4.1).

Of course, this expectation value is divergent in the UV, so it must be regulated. In [2] we showed how to carry out the regularization in detail, but for now it will be enough to compute the difference between the quenched expectation value and that for a fixed mass $m$ to produce a finite result. After subtracting, we get the finite difference

$$
\left\langle\phi^{2}\right\rangle_{\text {quench }}-\left\langle\phi^{2}\right\rangle_{\text {fixed }}=\frac{m^{2}}{4 \pi} \int \frac{d k}{k \sqrt{k^{2}+m^{2}}} \sin ^{2} k t
$$

for $d=3$. Interestingly, this expression can be integrated analytically and the solution expressed in terms of generalized hypergeometric functions,

$$
\left\langle\phi^{2}\right\rangle_{\text {quench }}-\left\langle\phi^{2}\right\rangle_{\text {fixed }}=\frac{m^{2} t^{2}}{4 \pi}\left(\frac{\pi}{2 t}{ }_{1} F_{2}\left(\frac{1}{2} ; 1, \frac{3}{2} ; m^{2} t^{2}\right)-{ }_{2} F_{3}\left(1,1 ; \frac{3}{2}, \frac{3}{2}, 2 ; m^{2} t^{2}\right)\right) .
$$




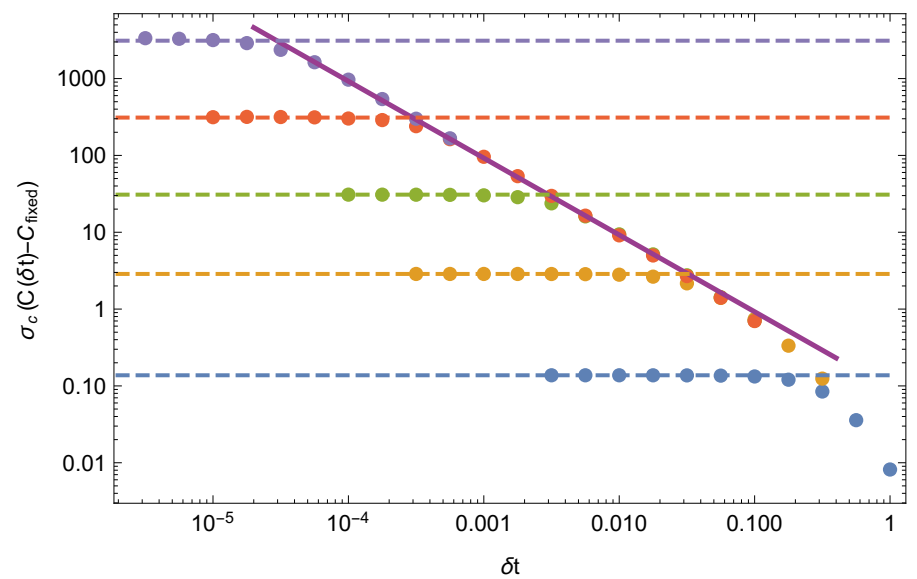

- $r=1$

$r=\frac{1}{10}$

$r=\frac{1}{10^{2}}$

$r=\frac{1}{10^{3}}$

$r=\frac{1}{10^{4}}$

(a) $\tau=1 / 4$

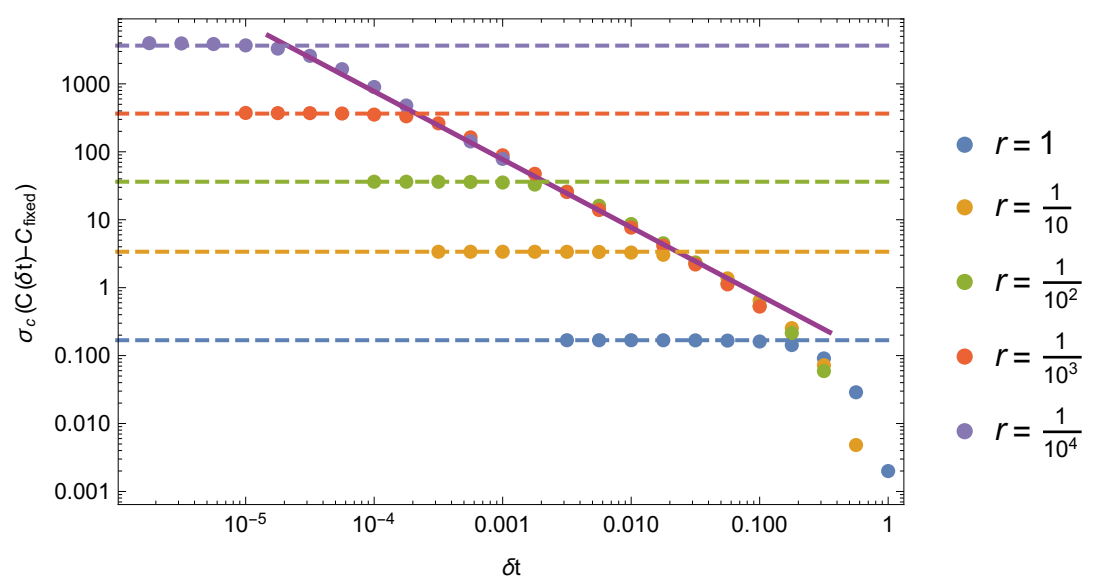

(b) $\tau=1 / 2$.

Figure 7. (Colour online) Spatial correlator under a smooth quench for fixed $t / \delta t=\tau$ as a function of both $\delta t$ and the distance separation $r$. In each case, we are subtracting the fixed mass correlator with $m^{2}(t / \delta t=\tau)$. The dashed lines correspond to computing the fixed mass quench correlator with $m=m_{\text {in }}$ for the different separations $r$. The purple solid line shows the analytic leading order contribution to $\left\langle\phi^{2}\right\rangle$, given by eq. (4.1).

The complete analysis of this solution can be found in [2], but let us just say here that the expectation value begins by growing linearly when $m t \ll 1$ (but still $t / \delta t \gg 1$ ) but then for very late times, i.e., $m t \gg 1$, the expectation value keeps increasing but now only at a logarithmic rate, i.e., $\left\langle\phi^{2}\right\rangle_{\mathrm{ren}} \sim \log (m t)$. As we show in [2], the instantaneous quench and the smooth quench calculation coincide for $d=3$, basically because the integrand for the smooth quench decays fast enough, in a way that the approximation of $\omega \delta t \ll 1$ continues to be valid. Recall from the discussion at the end of section 2, that it is possible to obtain the instantaneous quench expectation value starting from the smooth quench and taking both the late time limit, i.e., $t / \delta t \gg 1$, and the low energy limit, i.e., $\omega \delta t \ll 1$, for every $\omega$ in the problem. However, we generally need to integrate momentum $k$ up to infinity with fixed $\delta t$, so usually this approximation will break down for large enough $k$ (remember that 
$\omega_{\text {out }}=k$ in the quench to the critical point). In the special case of $d=3$, though, the integrand decays in a way that only the low momentum modes contribute and then the approximation is reasonable. In the next subsection, we will show that this is a particular effect of the three-dimensional case and does not hold in higher dimensional spacetimes.

\subsection{Higher dimensions}

The main problem that arises in taking the late time limit in higher dimensions is that the expectation value for the instantaneous quench cannot be regulated by simply subtracting the fixed mass expectation value for $d>3$. Moreover we will show that it cannot be regulated using the usual counterterms found in $[1,2]$. This fact will be taken as a hint to argue that in fact, the low energy approximation is not valid in evaluating the late time expectation value of $\phi^{2}$ in higher dimensions. Then, in order to get the expectation value for the smooth quench, what one should really do is to fully evaluate eq. (2.13) in the limit where $t / \delta t \gg 1$. Of course, without taking the extra low energy approximation, it will be impossible to recover the instantaneous quench answer, that it will turn out to be UV divergent for $d \geq 7$ and then, infinitely different from the UV finite result for the smooth quench.

So, let us start by evaluating the bare expectation value for $\phi^{2}$ in the case of an instantaneous quench. In this case we have,

$$
\left\langle\phi^{2}\right\rangle=\frac{\Omega_{d-2}}{2(2 \pi)^{d-1}} \int \frac{k^{d-4} d k}{\sqrt{k^{2}+m^{2}}}\left(k^{2}+m^{2} \sin ^{2}(k t)\right) .
$$

To explore the UV behaviour, we expand the expression above for large $k$ and for up to $d=9$, the results can be summarized as

$$
\begin{aligned}
\left\langle\phi^{2}\right\rangle=\frac{1}{\sigma_{s}} \int & d k k^{d-4}\left(k-\frac{m^{2}}{2 k}+\frac{3 m^{4}}{8 k^{3}}-\frac{5 m^{6}}{16 k^{5}}+O\left(k^{-7}\right)+\right. \\
& \left.+\sin ^{2}(k t)\left(\frac{m^{2}}{k}-\frac{m^{4}}{2 k^{3}}+\frac{3 m^{6}}{8 k^{5}}-\frac{5 m^{8}}{16 k^{7}}+O\left(k^{-9}\right)\right)\right) .
\end{aligned}
$$

Of course, the terms appearing in the first line are those same divergent terms that we expect from the constant mass case,

$$
\left\langle\phi^{2}\right\rangle_{\text {fixed }}=\frac{1}{\sigma_{s}} \int \frac{k^{d-2}}{\sqrt{k^{2}+m^{2}}} d k .
$$

But in eq. (5.4), we also have divergent terms in the second line proportional to $\sin ^{2}(k t)$ that do not correspond to any physical counterterm contributions found in eq. (3.11). In fact, as we are interested in the long time behaviour of the expectation value and given that the mass profile (and its time derivatives) decay exponentially in time, the only remaining physical counterterm in this limit should be the mass independent term of eq. (3.11), i.e., $k^{d-3}$.

In evaluating $\left\langle\phi^{2}\right\rangle_{\text {ren }}$, we integrate over all momenta, but the integral is divergent (even after taking into account the physical counterterms). The reason for this behaviour is that in higher dimensions the approximations of eq. (2.18) are not longer valid. One way to 
realize this is to compare it with the expression before that approximation. Recall that this is given by eq. (2.13), which after some manipulation, in the late time limit becomes,

$$
\begin{aligned}
\left\langle\phi^{2}\right\rangle_{\text {smooth }} & =\sigma_{s}^{-1} \int \Phi^{2}(k) d k \\
& =\sigma_{s}^{-1} \int d k\left(\frac{k^{d-2}}{\omega_{\text {out }}}\left\{\left|\alpha_{\vec{k}}\right|^{2}+\alpha_{\vec{k}} \beta_{-\vec{k}}^{\star} e^{2 i \omega_{\text {out }} t}+\alpha_{\vec{k}}^{\star} \beta_{\vec{k}} e^{-2 i \omega_{\text {out }} t}+\left|\beta_{\vec{k}}\right|^{2}\right\}-k^{d-3}\right),
\end{aligned}
$$

where $\alpha_{\vec{k}}$ and $\beta_{\vec{k}}$ are given by eq. (2.10). Now we wish to compare this integral with the result for an instantaneous quench

$$
\left\langle\phi^{2}\right\rangle_{\text {instant }}=\sigma_{s}^{-1} \int \Phi^{2}(k) d k=\sigma_{s}^{-1} \int d k\left(\frac{k^{d-4}}{\sqrt{k^{2}+m^{2}}}\left(k^{2}+m^{2} \sin ^{2}(k t)\right)-k^{d-3}\right) .
$$

For this comparison, we start by examining the integrands $\Phi^{2}(k)$. For $d=5$, this is done in figure 8 , where we choose $\delta t=10^{-3}$ and we evaluate the expression at $t=10$. However, our results generalize to the full range of values where the approximation of late times is valid. What we see is interesting: if we focus on small momenta, as shown in figure 8a, both the approximate and the full integrands agree. They both show a highly oscillating behaviour, that seems to continue to larger momenta and which would make both integrals diverge if it did so. However, what we see in figure $8 \mathrm{~b}$ is that in fact this behaviour does not continue for very large $k$ in the case of eq. (5.6). It can be seen that $\Phi^{2}(k)$ decays to zero for large momentum in the case of the full integral, as required to produce a UV finite result. Instead, the approximate integrand continues to oscillate and so produces a UV divergent integral. In figure 8 , we see that the two integrands differ substantially for $k \gtrsim 1000$, i.e., for $k \delta t \gtrsim 1$. Of course, the approximation of $\omega_{\text {out }} \delta t \ll 1$ is no longer valid in this regime and hence it is natural to expect that they should differ there. The approximations of eq. (2.18) are not valid to obtain the correct late time limit of $\left\langle\phi^{2}\right\rangle$ in higher dimensions and hence the expectation value does not match that after an instantaneous quench. To complete this analysis we show that the same happens for $d=7$ in figure 9 , where the oscillatory behaviour is even increased by a power law divergence in the approximate integrand.

To conclude this section, let us summarize our findings with regards to the late time limit after the quench. We have considered two different protocols to quench a scalar field: the instantaneous one, where we suddenly start evolving an eigenstate of the massive case with the massless Hamiltonian; and the smooth one, where we continuously evolve the mass of the scalar field from the massive case to the massless in a time scale of $\delta t$. Now one would think that these different protocols must give different answers in the early time evolution, ${ }^{7}$ but that in the limit of $\delta t$ going to zero and for late times, we should obtain similar results. In [2] we showed that effectively, if we take the limit of $t / \delta t \gg 1$ and also $\omega \delta t \ll 1$ for every $\omega$, we can reproduce the instantaneous quench result from that for the smooth quench and so in principle, we might expect the same late time behaviour in both cases. This allowed us to identify the interesting logarithmic growth behaviour of the scalar field for late times in $d=3$. In a self consistent way, we showed that both of these

\footnotetext{
${ }^{7}$ In our case, we obtain this interesting universal scaling near $t=0$.
} 


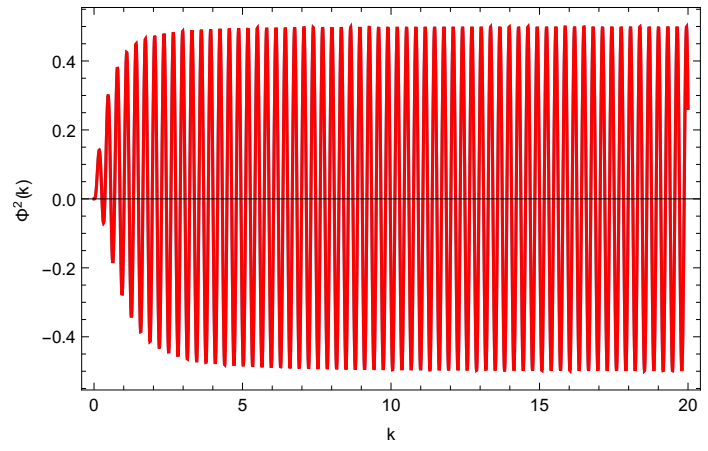

(a) $\Phi^{2}(k)$ for small momenta.

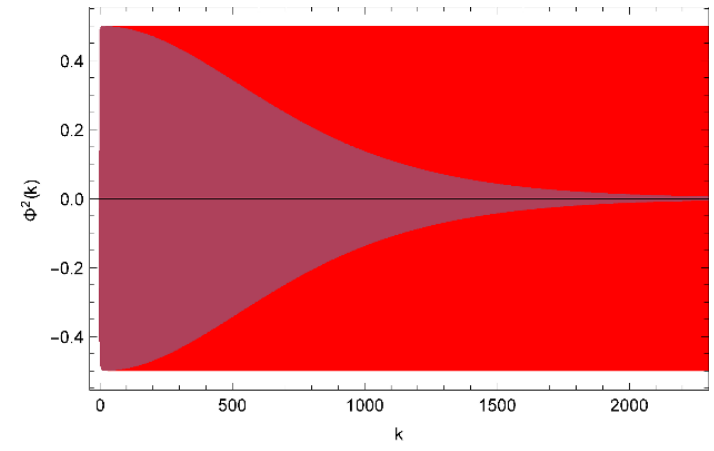

(b) $\Phi^{2}(k)$ for large momenta.

Figure 8. (Colour online) Analysis of the approximation of low energies and late times in $d=5$. We show results for $\delta t=10^{-3}, t=10$ and $m=1$. In the first figure, we show that $\Phi^{2}(k)$ in both eqs. (5.6) and (5.7) coincide when evaluated for small $k$. However in the second plot, we see that the full integrand (blue) decays for large $k$ while the approximate solution (red) keeps oscillating. The second plot looks fully painted because of the highly oscillatory nature of the functions.

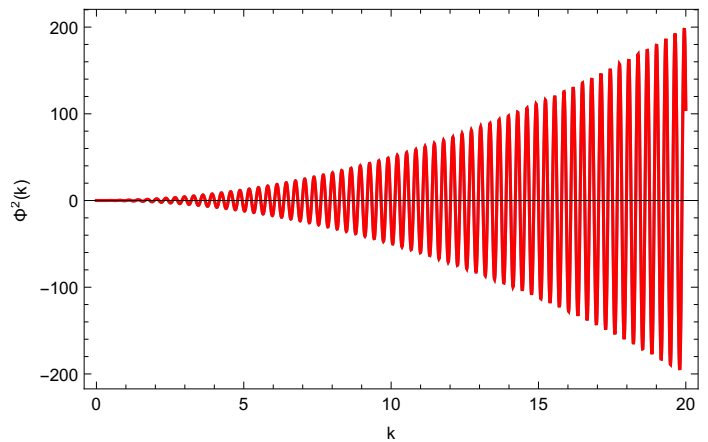

(a) $\Phi^{2}(k)$ for small momenta.

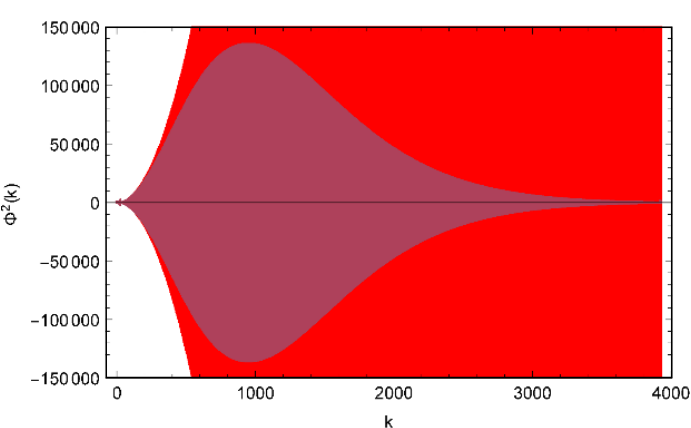

(b) $\Phi^{2}(k)$ for large momenta.

Figure 9. (Colour online) Analysis of the approximation of low energies and late times in $d=7$. We show results for $\delta t=10^{-3}, t=10$ and $m=1$. In the first figure, we show that $\Phi^{2}(k)$ of both eqs. (5.6) and (5.7) coincide when evaluated for small $k$. However in the second plot, we see that the full integrand (blue) decays for large $k$ while the approximate solution (red) keeps oscillating. The second plot looks fully painted because of the highly oscillatory nature of the functions.

approximations were reasonable in $d=3$ and so, the late time behaviour for the smooth and the instantaneous quench agreed. However, we found that this agreement in $d=3$ was fortuitous because when we tried to repeat the analysis in higher dimensions, we found that the approximations of eq. (2.18) are no longer valid. That is, higher momenta (and hence, higher frequencies) contribute significantly to the expectation value of $\phi^{2}$ in higher dimensions and cannot be neglected. Hence for $d \geq 4$, the instantaneous quench gives a result for $\left\langle\phi^{2}\right\rangle$ at late times which is infinitely different from the smooth quench result. In particular, the smooth quench gives a finite late time limit for $\left\langle\phi^{2}\right\rangle$ as $\delta t \rightarrow 0$ [2], while the corresponding result appears to diverge in an instantaneous quench. 


\subsection{Regulated instantaneous quench}

It is interesting to note that the integrand in eq. (5.7) for the instantaneous quench in higher dimensions does not decay to zero for large momentum. Instead, it seems to show a rapid oscillatory behaviour around zero, as shown in figures 8 and 9. So one may think that even though the amplitude is diverging, the positive and negative part are cancelling in every period and so, in some sense, we may be able to recover a finite result from these integrals. In fact, we are inspired here by the way that the fixed mass correlators were regulated in appendix A.

Hence let us go back to our instantaneous quench results. For $d=5$, the behaviour of the expectation value of $\phi^{2}$ for large $k$ can be extracted from eq. (5.4). This gives,

$$
\left\langle\phi^{2}\right\rangle \underset{k \rightarrow \infty}{\longrightarrow} \frac{1}{\sigma_{s}} \int d k\left(k^{2}-\frac{m^{2}}{2}+m^{2} \sin ^{2}(k t)\right) .
$$

The first divergence, proportional to $k^{d-3}$ is our usual counterterm that we will subtract. But note, then, that the term proportional to $\sin ^{2}$ can be re-written to yield

$$
\left\langle\phi^{2}\right\rangle_{\text {ren }} \underset{k \rightarrow \infty}{\longrightarrow} \frac{1}{\sigma_{s}} \int d k\left(-\frac{m^{2}}{2}+m^{2} \frac{1-\cos (2 k t)}{2}\right)=-\frac{1}{\sigma_{s}} \int d k \frac{\cos (2 k t)}{2},
$$

and this is one of the integrals that we now know how to regulate (just put $\alpha=0$ and $x=2 t$ in eq. (A.4). Of course, this is just showing the large $k$ behaviour of the integral. In order to get the full answer we should include all momenta and this, unfortunately, can only be done numerically. But, in principle, since we know how the integral behaves for large $k$, we should be able to get a finite result for our integral as well by using this new regulator. The results are shown in figure 10, where we evaluate numerically the instantaneous quench solution of eq. (5.7) for $d=5$ and adding a regulator $\exp (-a k)$,

$$
\left\langle\phi^{2}(a)\right\rangle_{\text {instant }}=\sigma_{s}^{-1} \int d k\left(\frac{k}{\sqrt{k^{2}+m^{2}}}\left(k^{2}+m^{2} \sin ^{2}(k t)\right)-k^{2}\right) \exp (-a k) .
$$

We evaluate the expectation value at late times, $m t=10$, and compare it with the smooth quench also at late times, where we are using $\delta t=1 / 20$. We find that as we take $a$ to zero, the regulated integral for the instantaneous quench approaches some finite value, showing that the integral converges. However, this value differs from that for the smooth quench in a relative amount by

$$
\left|\frac{\left\langle\phi^{2}(a \rightarrow 0)\right\rangle_{\text {instant }}-\left\langle\phi^{2}\right\rangle_{\text {smooth }}}{\left\langle\phi^{2}\right\rangle_{\text {smooth }}}\right| \sim\left|\frac{0.1670-0.1490}{0.1490}\right| \sim 0.121 .
$$

So even if we found a way to make sense of the divergent integral in $d=5$, the result does not quite coincide with the smooth quench result, where no approximation is made (apart from the late time limit approximation).

Note that the relative difference in eq. (5.11) is of order $m \delta t$. To quantify this difference more precisely we compute the expectation value in the instantaneous quench case with $a=10^{-3}$ and $m t=10$. We then vary $\delta t$ in the case of the smooth quench and compute the 


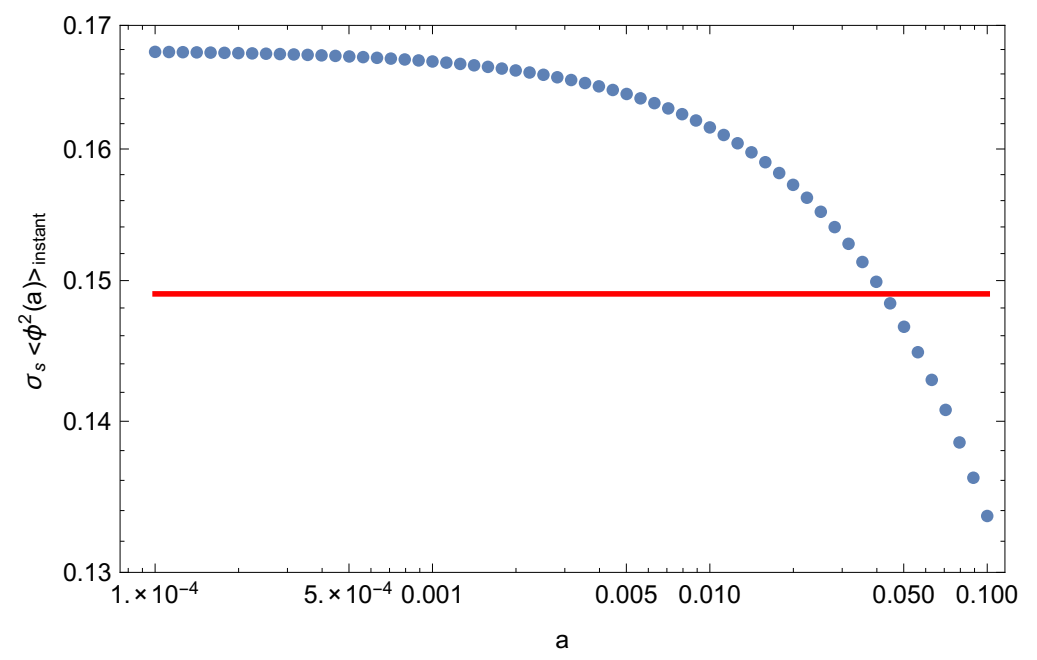

Figure 10. (Colour online) Expectation value of $\phi^{2}$ in the instantaneous quench as a function of the regulator parameter $a$ for $d=5$ and $m t=10$. The values of $a$ go from $10^{-1}$ to $10^{-4}$ in a logarithmic scale. The solid line represents the value for the smooth quench at $m t=10$ and $\delta t=1 / 20$.

relative error between the two results. The outcome is shown in figure 11 . We see that for $\delta t \sim 1$, the two protocols give very different answers. But this is expected because a large $\delta t$ means going into the adiabatic regime and this need not to agree with the rapid quench even at late times. Instead, as we decrease $\delta t$, we see that the relative difference between the two approaches also diminishes and in fact, when $\delta t$ is of order $10^{-3}$, the relative error is also of that order of magnitude.

Naively, one may think that it is possible to understand this behaviour by expanding the expectation value in powers of $\delta t$. To do that we start with the smooth quench integral in eq. (5.6). We then expand the Bogoliubov coefficients $\alpha_{\vec{k}}$ and $\beta_{\vec{k}}$ for small $\delta t$ and compute the integrand to lowest orders in $\delta t$. This results in

$$
\begin{aligned}
\left\langle\phi^{2}\right\rangle_{\text {smooth }}=\frac{1}{\sigma_{s}} \int d k & \frac{k^{d-4}}{\sqrt{k^{2}+m^{2}}}\left(\left(k^{2}+m^{2} \sin (k t)^{2}\right)+\right. \\
& \left.+\delta t^{2} \frac{\pi^{2} m^{2}}{12}\left(k^{2}-\left(2 k^{2}+m^{2}\right) \sin ^{2}(k t)\right)+O\left(\delta t^{3}\right)\right) .
\end{aligned}
$$

Let us analyse this last expression. The first term is independent of $\delta t$ and one can easily see that it exactly matches the expression for the instantaneous quench in eq. (5.7). Of course, this match was known implicitly, since expanding for $\delta t \ll 1$ in the previous expression is actually expanding for $\omega \delta t \ll 1$ and the agreement found above is the claim that we could reproduce the instantaneous result by taking the small frequency limit of the smooth quench. The next term in the $\delta t$ expansion appears at order $\delta t^{2}$. So, again naively, one might expect that $\left\langle\phi^{2}\right\rangle_{\text {smooth }}=\left\langle\phi^{2}\right\rangle_{\text {instant }}+\gamma \delta t^{2}+O\left(\delta t^{3}\right)$, for some number $\gamma$. However, if we look carefully at the integral that gives this correction at order $\delta t^{2}$, we will see that it is in fact divergent for $d \geq 5$. We can try to regulate it by adding a regulator as in appendix A, but in fact we will see that apart from the oscillating term (which can be 


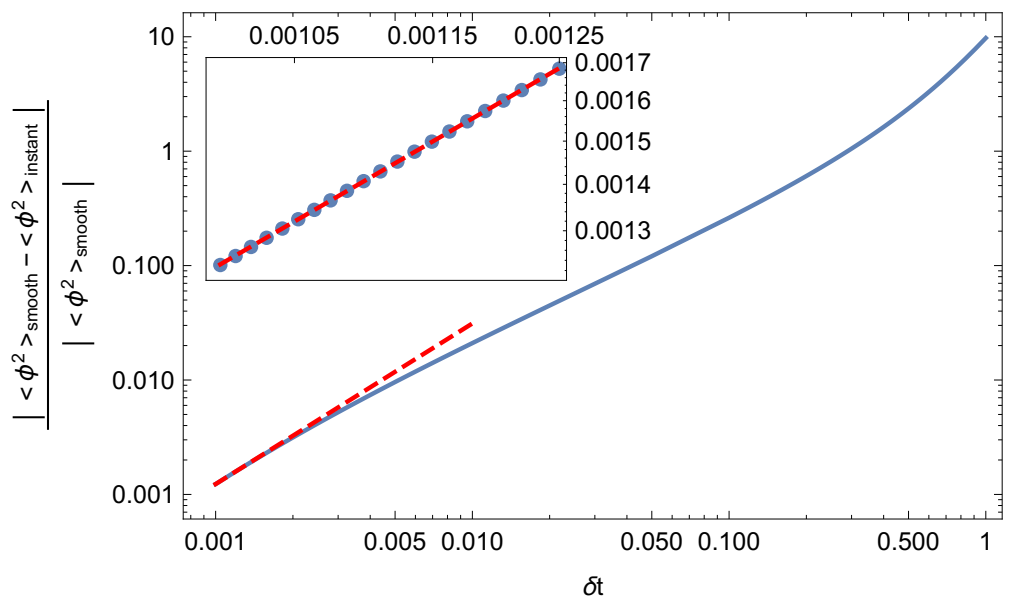

Figure 11. (Colour online) Relative error in the computation of $\left\langle\phi^{2}\right\rangle$ at late times using the smooth and the instantaneous quench as a funtion of $\delta t$. Here we are regulating the instantaneous result with $a=10^{-3}$ and evaluating at time $m t=10$. The red dashed line is showing the fit of the first points (from $\delta t=1 / 1000$ to $\delta t=1 / 800$ ) by a power law function of the type $f(\delta t)=a \delta t^{\alpha}$, where $a=20.03$ and $\alpha=1.404$. The inset zooms in the region where the fit was made showing perfect agreement between the points and the fit.

regulated) there is an extra constant term in the integrand, i.e., $-\frac{1}{24 \sigma_{s}} \pi^{2} \delta t^{2} m^{4}$, that will make the integral divergent as we integrate over $k$ from 0 to $\infty$.

This is closely related to the fact that if we try to fit the relative error by some power law expression in the region of small $\delta t$ - see red dashed line in figure 11 - we find that the error does not scale as $\delta t^{2}$ but the exponent is rather close to 1.40. This is another sign that this naive expansion is somehow ill-defined for $d=5$. Again, we should say that behind this expansion it is assumed not only that $\delta t$ is small but that $\omega \delta t \ll 1$, for every $\omega$ in the problem, and we already showed that the assumption is not valid for $d \geq 5$.

The situation is even worse in higher dimensions, where the first term in the series, i.e., the order $\delta t^{0}$ term in eq. (5.12), fails to converge. As an example, we show what happens in $d=7$. Even though in figure 9 the integrand appears to oscillate around zero, this is not the case. For large $k$, we have, after subtracting the usual counterterms,

$$
\begin{aligned}
\left\langle\phi^{2}\right\rangle_{\text {ren }} \underset{k \rightarrow \infty}{\longrightarrow} & \frac{1}{\sigma_{s}} \int d k\left(-\frac{m^{2}}{2} k^{2}+\frac{3 m^{4}}{8}+\left(m^{2} k^{2}-\frac{m^{4}}{2}\right) \sin ^{2}(k t)\right) \\
& \simeq \frac{1}{\sigma_{s}} \int d k\left(\frac{1}{4}\left(m^{4}-2 m^{2} k^{2}\right) \cos (2 k t)+\frac{m^{4}}{8}\right) .
\end{aligned}
$$

So, even though the first two terms could be regulated using the above prescription, there is an extra constant term that cannot. Adding the regulator to the term proportional to $m^{4} / 8$, we will get a result which diverges as $a \rightarrow 0$,

$$
\frac{m^{4}}{8} \int_{0}^{\infty} \exp (-a k)=\frac{m^{4}}{8 a},
$$

and so, the limit of $a \rightarrow 0$ in this case will be nonsense. The same also happens in any higher number of dimensions. In fact, the only reason why this worked in $d=5$ was 
because the divergent terms with the $\sin ^{2}$ term, exactly matched the terms without the $\sin ^{2}$ term, in a way that made the whole integrand to oscillate around zero. But this, as shown above, does not happen in general and so, the instantaneous quench approximation in higher dimensions gives a value for $\left\langle\phi^{2}\right\rangle$ which differs by an infinite amount from the smooth quench result, even in the late time limit.

\section{The energy density at late times}

In $[1,2]$, it was argued that the renormalized energy density for a quench satisfying eq. (1.2) obeys a scaling relation (1.3),

$$
\delta \mathcal{E}_{\text {ren }} \sim \delta \lambda^{2}(\delta t)^{d-2 \Delta} .
$$

This result is consistent with the scaling of the expectation value of the operator (1.4) since it satisfies the Ward identity

$$
\frac{d \mathcal{E}_{\mathrm{ren}}}{d t}=-\partial_{t} \lambda(t)\left\langle\mathcal{O}_{\Delta}\right\rangle_{\mathrm{ren}}
$$

In the corresponding scaling relation (1.4) for the quenched operator, $\left\langle\mathcal{O}_{\Delta}\right\rangle_{\text {ren }}$ is measured earlier than or soon after the end of the quench. However, the energy scaling (6.1) will be valid for arbitrarily late times since the energy is injected into the system only during the quench. The equation (6.1) gives the $\delta t$ dependence. The energy density itself will have additional finite pieces, which would be subdominant for $\Delta>d / 2$, but in fact give the dominant contribution for $\Delta<d / 2$.

In this section, we will concentrate on the energy density for the free bosonic field with the mass profile (2.2) at asymptotically late times and calculate a UV finite quantity: the excess energy above the ground state energy of the system with the value of the coupling at asymptotically late times. We will perform the $\delta t \rightarrow 0$ limit and compare the results with that of an instantaneous quench.

In terms of the "in" modes the energy density is given by

$$
\mathcal{E}=\frac{1}{2} \int \frac{d^{d-1} k}{(2 \pi)^{d-1}}\left(\left|\partial_{t} u_{\vec{k}}\right|^{2}+\left(k^{2}+m^{2}(t)\right)\left|u_{\vec{k}}\right|^{2}\right)
$$

Since we are interested in the behavior of this quantity at late times, it is convenient to express this in terms of the "out" modes. In terms of the Bogoliubov coefficients $\alpha_{\vec{k}}, \beta_{\vec{k}}$ defined in (2.9) and (2.10) this becomes

$$
\begin{aligned}
\mathcal{E}=\frac{1}{2} \int \frac{d^{d-1} k}{(2 \pi)^{d-1}} & \left(\left[\omega_{\text {out }}^{2}+k^{2}+m^{2}(t)\right]\left(\left|\alpha_{\vec{k}}\right|^{2}+\left|\beta_{\vec{k}}\right|^{2}\right)\left|v_{\vec{k}}\right|^{2} \mid\right. \\
& \left.+\left[k^{2}+m^{2}(t)-\omega_{\text {out }}^{2}\right]\left(\alpha_{\vec{k}} \beta_{\vec{k}}^{\star} v_{\vec{k}} v_{-\vec{k}}+\alpha_{\vec{k}}^{\star} \beta_{\vec{k}} v_{\vec{k}}^{\star} v_{-\vec{k}}^{\star}\right)\right) .
\end{aligned}
$$

In deriving this expression, we have used the fact that $\alpha_{\vec{k}}$ and $\beta_{\vec{k}}$ depend only on $|\vec{k}|$. At $t=\infty$, the second line of eq. (6.4) vanishes. Using the relation $\left|\alpha_{\vec{k}}\right|^{2}-\left|\beta_{\vec{k}}\right|^{2}=1$ and the asymptotic form of the out modes at $t \rightarrow \infty$, i.e.,

$$
v_{\vec{k}} \rightarrow \frac{1}{\sqrt{2 \omega_{\text {out }}}} e^{i\left(\vec{k} \cdot \vec{x}-\omega_{\text {out }} t\right)},
$$


one gets

$$
\mathcal{E}=\frac{1}{2} \int \frac{d^{d-1} k}{(2 \pi)^{d-1}} \omega_{\text {out }}\left(1+2\left|\beta_{\vec{k}}\right|^{2}\right) .
$$

However, the ground state energy of the system with the final value of the mass is given by

$$
\mathcal{E}_{\text {ground }}=\frac{1}{2} \int \frac{d^{d-1} k}{(2 \pi)^{d-1}} \omega_{\text {out }} .
$$

Therefore the excess energy over the final ground state is given by

$$
\Delta \mathcal{E}=\int \frac{d^{d-1} k}{(2 \pi)^{d-1}} \omega_{\text {out }}\left|\beta_{\vec{k}}\right|^{2}
$$

Using the explicit form of the Bogoliubov coefficients in eq. (2.10) and integrating over the angles, we arrive at the final expression

$$
\Delta \mathcal{E}=\frac{\Omega_{d-2}}{(2 \pi)^{d-1}} \int_{0}^{\infty} d k k^{d-2} \omega_{\text {out }} \frac{\sinh ^{2}\left(\pi \omega_{-} \delta t\right)}{\sinh \left(\pi \omega_{\text {in }} \delta t\right) \sinh \left(\pi \omega_{\text {out }} \delta t\right)}
$$

For the mass profile (2.2), the integral in eq. (6.9) is finite for any finite $\delta t$. In fact, for small $k$, the integrand approaches $\left(k^{d-2}\right) \frac{\tanh (\pi m \delta t)}{2 \pi \delta t}$, while for large $k$, it becomes $\left(k^{d-3}\right) m^{4}(\delta t)^{2} e^{-2 \pi k \delta t}$. Hence the integral above is convergent both in the IR and UV for any physical $d \geq 2$.

To analyze the small $\lambda=m \delta t$ limit of eq. (6.9), let us first scale out a power of $\delta t$ to write $\Delta \mathcal{E}=(\delta t)^{-d} I_{1}(\lambda)$ where

$$
I_{1}(\lambda)=\int_{0}^{\infty} d q q^{d-1} \frac{\sinh ^{2}\left[\frac{\pi}{2}\left(\sqrt{q^{2}+\lambda^{2}}-q\right)\right]}{\sinh \left(\pi \sqrt{q^{2}+\lambda^{2}}\right) \sinh (\pi q)} .
$$

Clearly $I_{1}(0)=0$. However the small $\lambda$ dependence is different for different dimensions. It turns out that

$$
\begin{aligned}
I_{1}(\lambda) & \sim \lambda^{d} \quad d=2,3, \\
& \sim \lambda^{4} \quad d \geq 4 .
\end{aligned}
$$

The above behavior was determined from a direct numerical evaluation of the integral in eq. (6.10) and fitting the results shown in figure 12.

This means that for $d=2,3$, the excess energy has a smooth finite small $\delta t$ limit with $\Delta \mathcal{E} \sim m^{d}$. The leading answer is exactly the same as the energy excess for the instantaneous quench, which can be read off easily from the corresponding Bogoliubov coefficients (2.16) (for the mass profile (2.2))

$$
\Delta \mathcal{E}^{\text {instant }}=\frac{\Omega_{d-2}}{(2 \pi)^{d-1}} \int d k k^{d-1} \frac{\left(\sqrt{k^{2}+m^{2}}-k\right)^{2}}{4 k \sqrt{k^{2}+m^{2}}} .
$$

This quantity $\Delta \mathcal{E}^{\text {instant }}$ is finite for $d=2,3$ and hence we have

$$
\left.\Delta \mathcal{E}^{\delta t \rightarrow 0}\right|_{d=2}=\frac{m^{2}}{16 \pi},
$$




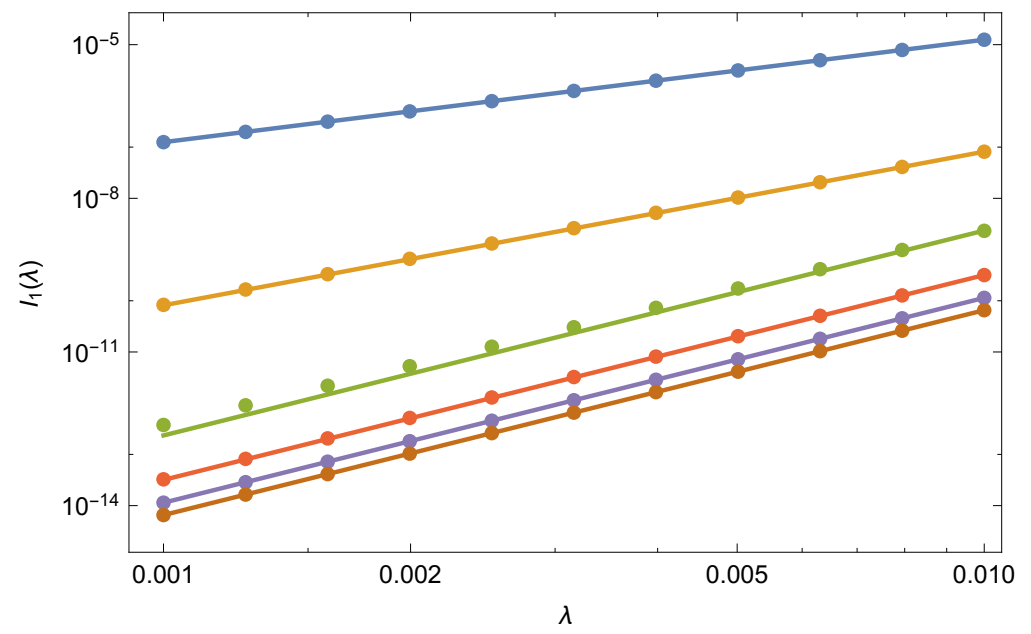

Figure 12. (Colour online) The integral $I_{1}(\lambda)$ in eq. (6.10) as a function of $\lambda$ on a logarithmic scale. The data are for $d=2,3,4,5,6,7$ from top to bottom. The solid lines correspond to $I_{1}(\lambda) \sim \lambda^{d}$ for $d=2,3$ and $I_{1}(\lambda) \sim \lambda^{4}$ for $d=4,5,6,7$. Note that the fit in $d=4$ is not as good as the others, which suggests that there are log corrections to the leading behavior for $d=4$.

$$
\left.\Delta \mathcal{E}^{\delta t \rightarrow 0}\right|_{d=3}=\frac{m^{3}}{24 \pi}
$$

To estimate the corrections to this leading small $\lambda$ behavior consider the difference of the excess energy to the excess energy due to an instantaneous quench,

$$
\delta \mathcal{E}=\Delta \mathcal{E}-\Delta \mathcal{E}^{\text {instant }}=\delta t^{-d}\left(I_{1}(\lambda)-\delta t^{d} \Delta \mathcal{E}^{\text {instant }}\right) \equiv m^{d} I_{2}(\lambda) .
$$

A numerical evaluation of this quantity shows

$$
\delta \mathcal{E} \sim m^{4} \delta t^{4-d}
$$

both for $d=2$ and 3. This behaviour is shown in figure 13, which is a log-log plot of the quantity $I_{2}(\lambda)$. Clearly, we have $I_{2}(\lambda) \sim \lambda^{2}$ for $d=2$ and $I_{2}(\lambda) \sim \lambda$ for $d=3$, which leads to the scaling in eq. (6.15).

On the other hand, for $d=4,5, \ldots$, one recovers the desired scaling from the leading behaviour, i.e.,

$$
\Delta \mathcal{E} \sim \lambda^{4} \delta t^{-d}=m^{4} \delta t^{4-d},
$$

with logarithmic corrections for even dimensions. Thus for these dimensions, the energy density diverges in the $m \delta t \rightarrow 0$ limit. At the same time, the energy density for an instantaneous quench diverges in the UV for these dimensions. Let us emphasize this point once again: our results show that the energy density is UV finite after a smooth quench; however, in the limit $m \delta t \rightarrow 0$, that energy density diverges, just as in the instantaneous quench, where the divergence is in the UV.

These results also indicate that for $d \geq 4$, the excess energy is in fact given by linear response. This is in accord with the computation of the renormalized expectation value of $\phi^{2}$ and the renormalized energy density for $d \geq 4$, as described in [1,2]. Indeed if we 


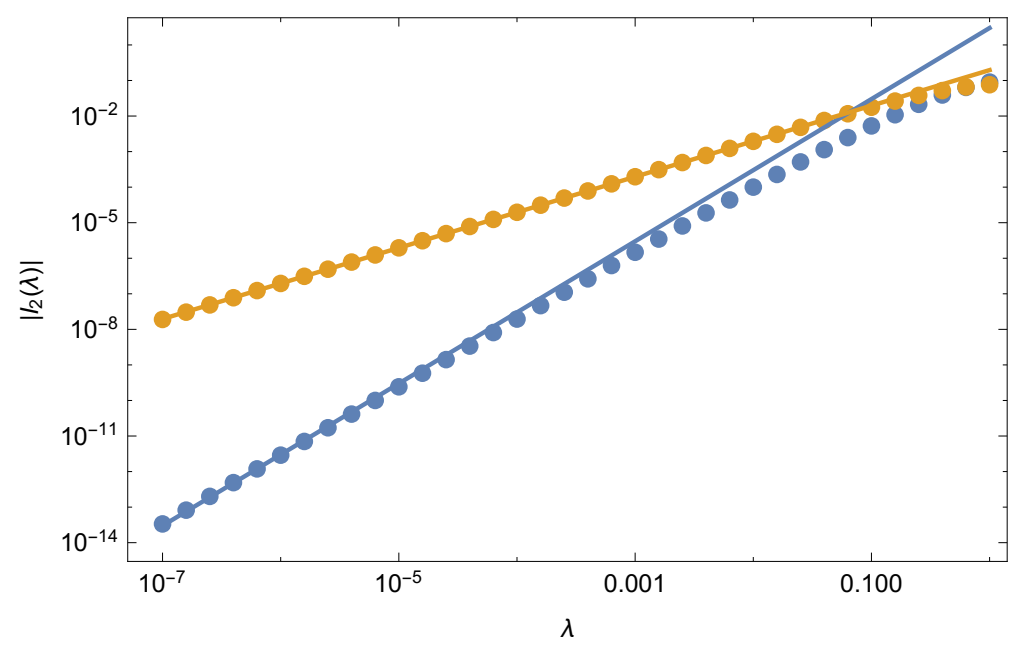

Figure 13. (Colour online) The absolute value of $I_{2}(\lambda)=m^{-d} \delta \mathcal{E}$ as a function of $\lambda=m \delta t$ on a logarithmic scale. The data are for $d=2,3$ from bottom to top. The solid lines correspond to the curves $I_{2}(\lambda) \sim \lambda^{2}$ for $d=2$ and $\lambda$ for $d=3$. Those curves correspond to the best fit of the numerical data in the region $10^{-7}<\lambda<10^{-5}$.

extract the leading piece in a small $\lambda$ expansion by expanding the integrand in eq. (6.10) we get the expression

$$
I_{1}(\lambda) \sim \frac{\pi^{2} \lambda^{4}}{16} \int_{0}^{\infty} d q \frac{q^{d-3}}{(\sinh (\pi q))^{2}}
$$

This expansion makes sense when the above integral is convergent. However the integral is IR divergent for $d=2,3,4$. The latter explains is why $I_{1}(\lambda)$ does not have an expansion in terms of $\lambda^{2}$ in these dimensions.

\section{$7 \quad$ Excess energy for general theories}

The discussion in section 6 suggests that the scaling of the excess energy should be a property of a general interacting field theory. In this section, we argue that this is indeed true.

Consider an action

$$
S=S_{C F T}+\int d t \lambda(t) \int d^{d-1} x \mathcal{O}(\vec{x}, t)
$$

where $S_{C F T}$ is a conformal field theory action. The function $\lambda(t)$ is of the form

$$
\lambda(t)= \begin{cases}\lambda_{0} & \text { for } t<0 \\ \lambda_{0}+\delta \lambda F(t / \delta t) & \text { for } 0 \leq t \leq \delta t \\ \lambda_{1}=\lambda_{0}+\delta \lambda & \text { for } t>\delta t\end{cases}
$$

Alternatively, we may write $\lambda(t)=\lambda_{0}+\delta \lambda F(t / \delta t)$ if we specify $F(y \leq 0)=0$ and $F(y \geq 1)=1$. We leave the details of the function $F(y)$ during the transition (i.e., $0 \leq y \leq 1$ ) unspecified other than that the maximum is finite with $F_{\max } \geq 1$. Further, this profile may dip below zero by some finite amount and so we specify the minimum as $F_{\min } \leq 0$. Implicitly, we are also assuming that the profile is smooth. 
The system is prepared in the ground state of the initial action. Let us evaluate the total energy density $\mathcal{E}(t)$ at some time $t>\delta t$ in a perturbation expansion in $\delta \lambda$. To quadratic order in $\delta \lambda$, this expression is given by

$$
\begin{aligned}
\mathcal{E}(t)= & \mathcal{E}_{0}-\delta \lambda F(t / \delta t)\left\langle 0, \lambda_{0}|\mathcal{O}(\overrightarrow{0}, 0)| 0, \lambda_{0}\right\rangle \\
& -i \delta \lambda^{2} F(t / \delta t) \int_{0}^{t} d t^{\prime} \int d^{d-1} x^{\prime} F\left(t^{\prime} / \delta t\right)\left\langle 0, \lambda_{0}\left|\left[\mathcal{O}\left(\vec{x}^{\prime}, t\right), \mathcal{O}\left(\overrightarrow{0}, t^{\prime}\right)\right]\right| 0, \lambda_{0}\right\rangle \\
& -\mathcal{E}_{0} \delta \lambda^{2} \int_{0}^{t} d t^{\prime} \int_{0}^{t^{\prime}} d t^{\prime \prime} \int d^{d-1} x^{\prime} F\left(t^{\prime} / \delta t\right) F\left(t^{\prime \prime} / \delta t\right)\left\langle 0, \lambda_{0}\left|\mathcal{O}\left(\vec{x}^{\prime}, t^{\prime}\right) \mathcal{O}\left(\overrightarrow{0}, t^{\prime \prime}\right)\right| 0, \lambda_{0}\right\rangle \\
& -\mathcal{E}_{0} \delta \lambda^{2} \int_{0}^{t} d t^{\prime} \int_{0}^{t^{\prime}} d t^{\prime \prime} \int d^{d-1} x^{\prime} F\left(t^{\prime} / \delta t\right) F\left(t^{\prime \prime} / \delta t\right)\left\langle 0, \lambda_{0}\left|\mathcal{O}\left(\overrightarrow{0}, t^{\prime \prime}\right) \mathcal{O}\left(\vec{x}^{\prime}, t^{\prime}\right)\right| 0, \lambda_{0}\right\rangle \\
& +\delta \lambda^{2} \int_{0}^{t} d t^{\prime} \int_{0}^{t} d t^{\prime \prime} \int d^{d-1} x^{\prime} F\left(t^{\prime} / \delta t\right) F\left(t^{\prime \prime} / \delta t\right)\left\langle 0, \lambda_{0}\left|\mathcal{O}\left(\vec{x}^{\prime}, t^{\prime}\right) H_{0} \mathcal{O}\left(\overrightarrow{0}, t^{\prime \prime}\right)\right| 0, \lambda_{0}\right\rangle \\
& +O\left(\delta \lambda^{3}\right),
\end{aligned}
$$

where $H_{0}$ is the initial Hamiltonian, $\left|0, \lambda_{0}\right\rangle$ is the ground state of the initial Hamiltonian, and $\mathcal{E}_{0}$ denotes the initial ground state energy density. Here we have used space translation invariance as well as the fact that one point functions in the initial ground state are constants in both space and time. On the other hand, the expectation value of the operator $\mathcal{O}$ is, to order $\delta \lambda$, given by

$$
\left\langle 0, \lambda_{0}|\mathcal{O}(\vec{x}, t)| 0, \lambda_{0}\right\rangle=\left\langle 0, \lambda_{0}|\mathcal{O}(\overrightarrow{0}, 0)| 0, \lambda_{0}\right\rangle-i \int d^{d-1} x \int_{0}^{t} d t^{\prime} \lambda\left(t^{\prime}\right)\left\langle 0, \lambda_{0}\left|\left[\mathcal{O}(\vec{x}, t), \mathcal{O}\left(\overrightarrow{0}, t^{\prime}\right)\right]\right| 0, \lambda_{0}\right\rangle .
$$

Using this, it is straightforward to verify that the Ward identity

$$
\frac{d \mathcal{E}(t)}{d t}=-\frac{d \lambda(t)}{d t}\left\langle 0, \lambda_{0}|\mathcal{O}(\vec{x}, t)| 0, \lambda_{0}\right\rangle
$$

is satisfied. ${ }^{8}$

We are interested in evaluating eq. (7.2) at late times. However since the coupling is a constant for $t \geq \delta t$, the energy density at infinitely late times is exactly the same as the energy density at $t=\delta t$. The ground state energy density of the final Hamiltonian is given by the standard expression

$$
\mathcal{E}_{f}=\mathcal{E}_{0}-\delta \lambda\left\langle 0, \lambda_{0}|\mathcal{O}(\overrightarrow{0}, 0)| 0, \lambda_{0}\right\rangle-\frac{\delta \lambda^{2}}{V^{2}} \sum_{n \neq 0} \frac{\left|\left\langle 0, \lambda_{0}\left|\int d^{d-1} x \mathcal{O}(\vec{x}, 0)\right| n, \lambda_{0}\right\rangle\right|^{2}}{\mathcal{E}_{0}-\mathcal{E}_{n}}+\cdots,
$$

where $\mathcal{E}_{n}$ denote the energy densities of the excited states $\left|n, \lambda_{0}\right\rangle$ of the initial Hamiltonian and $V$ is the volume of the system. It is clear from eqs. (7.2) and (7.5) that the excess energy density $\Delta \mathcal{E}=\mathcal{E}(\delta t)-\mathcal{E}_{f}$ starts at $O\left(\delta \lambda^{2}\right)$. Moreover we expect that the UV divergences in $\mathcal{E}(t)$ for $t \geq \delta t$ are cancelled by those in $\mathcal{E}_{f}$. This expectation comes from the following fact: as seen in the previous sections, and in [1, 2], the UV divergent terms

\footnotetext{
${ }^{8}$ The time derivatives of the second and third lines in eq. (7.2) cancel the time derivatives which act on the upper limit of integration in the third term of the first line.
} 
depend on both $\lambda(t)$ and its time derivatives. However for $t>\delta t$, the coupling is constant and these time derivatives vanish. Therefore, the UV divergent terms should be those of the constant coupling interacting theory. While this is explicit in the free field theory considered in section 6 , we do not have an explicit proof for general interacting theories but it stands as a reasonable expectation. If the resulting expression for $\Delta \mathcal{E}$ is also IR finite, it is determined to this order entirely by dimensional analysis,

$$
\Delta \mathcal{E} \sim \delta \lambda \delta t^{d-2 \Delta}
$$

Further, as discussed in $[1,2]$, the corrections to this result would be a power series in the dimensionless coupling $g=\delta \lambda \delta t^{d-\Delta}$, which is small in the fast quench limit.

This argument will fail if the integrals involved in $\Delta \mathcal{E}$ are IR divergent. This can be seen to happen when $2 \Delta>d$, as we have explicitly seen for the free field theory for $d=2$ and 3 .

\section{Discussion}

The aim of this paper is to establish a precise relation between the smooth fast quench and the instantaneous quench. These are the most common quench protocols discussed in high energy theory and condensed matter physics literature, respectively. Naive reasoning would say that if one considers the evolution at very late times (with respect to the quench rate $\delta t$ ), then both protocols should give the same result since $\delta t$ would be negligible. However, our results in this paper suggest that they may or may not give the same answers depending on a variety of factors such as the spacetime dimension, the scaling dimension of the quenched operator and how much time after the quench is considered. In this paper, we computed spatial correlators, local expectation values and the energy density. In this section, we summarize our results, making precise statements on when the abrupt approximation makes sense. We also discuss different procedures to regulate the energy density. The bulk of our comments below relate to the explicit calculations performed in the free field theory. However, as we will discuss at the end of this section, we expect that these conclusions hold for generic interacting theories for smooth fast quenches as defined in eq. (1.2).

Spatial correlators. The study of spatial two-point correlation functions is interesting because they are UV-finite quantities that introduce a new scale to the problem, i.e., the spatial separation $r$. The behaviour of this object is very different depending on the time at which we compute it. We summarize the results here for early times, i.e., $t / \delta t \sim O(1)$, and for very late times, i.e., $t / \delta t \gg 1$ and $m t \gg 1$.

- At early times, we can distinguish between three different regimes.

When $m \delta t>1$, independently of $r$, we are in the slow quench regime, so we cannot compare with our previous results. However, there should be some signatures of universal behaviour corresponding to the Kibble-Zurek scaling when quenching through a critical point. We leave this appealing point for further research in the future [28].

The most interesting feature appears when $r<\delta t<1 / m$. This means that the quench is fast since $m \delta t<1$ but the spatial separation defines the smallest scale. 
Then we found that the same universal scaling that was reported in $[1,2]$ appears in the two-point spatial correlation function. This holds in any spacetime dimension and for arbitrary "early" times. We can think of this correlator as a version of $\left\langle\phi^{2}\right\rangle$ regulated with point splitting and so, $r$ plays the role of a short distance cut-off see below.

In general, we would continue to decrease $\delta t$ towards the instantaneous limit in which $\delta t \rightarrow 0$. However, in this correlator, we are limited by the distance separation $r$. In fact, the correlator saturates as $\delta t$ gets of order $r$ and then the result becomes independent of $\delta t$. In all this analysis we were able to take the UV cut-off to infinity but we expect a similar behaviour when working in theories with a finite cut-off, with $r^{-1}$ playing that role here.

- At late times, the results depend on the spacetime dimensions and on the separation distance. For long distances, in any dimensions, the correlator for the instantaneous quench and the smooth quench coincide. As the separation becomes smaller, the behaviour is different depending on the spacetime dimensions: for $d=3$, the smooth and the instantaneous correlator continue to coincide as $r \rightarrow 0$; for $d=5$, there appears a small finite difference that goes to zero as $\delta t$ goes to zero; finally, for $d=7$ the two correlators differ by an infinite amount as $r \rightarrow 0$. We expect the latter behaviour extends in higher dimensions.

Expectation value of $\phi^{2}$. We showed that the short distance expansion of the correlator is in one-to-one correspondence with the counterterms needed to regulate the bare expectation value of $\phi^{2}$. Then, it should not be a surprise that the behaviour of the $\left\langle\phi^{2}\right\rangle$ at late times is very similar to that of the spatial two-point correlator at late times but with small spatial separation. In fact, we showed that for $d=3$, the smooth and the instantaneous quench give the same answer. When evaluated for $d=5$, the smooth quench differs from the instantaneous quench but only in a finite amount that is of order $\delta t$. In higher dimensions, however, it is impossible to regulate the expectation value of $\phi^{2}$ in the case of the instantaneous quench. The smooth quench, in contrast, has a smooth finite limit as $\delta t \rightarrow 0$ and so, the two approaches yield infinitely different results.

Regulating the energy density. Both in the present and previous [1,2] works, we worked in a framework where UV-divergent quantities where regulated by adding suitable counterterms. Of course, we showed how to construct those counterterms and how they yield finite values for quantities such as the expectation value of $\left\langle\phi^{2}\right\rangle$ and the energy density. The way in which such a subtraction is done is much in the spirit of how regularization works in AdS/CFT through what is known as holographic renormalization [29-31]. There we add an extra counterterm (boundary) action to the usual gravitational action to get finite expectation values. This is how, for instance, expectation values for holographic quantum quenches are regulated in [23, 24], which served as a motivation to our studies. Our approach is also reminiscent of the way field theories in curved spacetimes are regulated.

However, a number of things about this procedure may appear strange to a typical field theorist. In particular, the fact that our counterterms first, depend on time and second, 
some terms depend on time derivatives of the quenched coupling. In this section, we would like to go back to this procedure and compare it with other candidates.

To summarize, we define a renormalized energy density by subtracting counterterm contributions from a bare energy density (and taking the cut-off to infinity). Basically, $\langle\mathcal{E}\rangle_{\text {ren }} \equiv \mathcal{E}_{\text {quench }}-\mathcal{E}_{c t}$ where $\mathcal{E}_{\text {quench }}$ and $\mathcal{E}_{c t}$ separately diverge as the cut-off goes to infinity but $\langle\mathcal{E}\rangle_{\text {ren }}$ is finite. We also showed in [2] that with this definition of renormalized energy density (and an equivalent for the scalar field), we satisfy the Ward identity in any spacetime dimension and for any quench protocol.

A second, perhaps more standard approach, would be to recognize the divergences as coming from the zero-point energy for the scalar field. Each momentum mode behaves as a single harmonic oscillator and then if we sum all the zero-point energies, i.e., $\frac{1}{2} \omega(k)$, we get a divergent quantity. This is what we call $\mathcal{E}_{\text {fixed }}$, that is, the energy density for a scalar field of fixed mass at any instant of time. Again, one would naively say that we should get a UV finite value if we subtract $\mathcal{E}_{\text {quench }}-\mathcal{E}_{\text {fixed }}$. One nice thing about this is that if we go to very early or very late times where the mass is constant, there is a ground state and it has precisely zero energy density and any other state has a positive energy density. Even if this procedure works for low dimensional spacetimes, we showed that is not enough in higher dimension. In fact, for $d=3$ and 5 , we have

$$
\mathcal{E}_{\text {fixed }}-\mathcal{E}_{c t} \propto m^{d}
$$

while in $d=7$,

$$
\mathcal{E}_{\text {fixed }}-\mathcal{E}_{c t} \propto m^{7}+m^{2} \partial_{t}^{2} m^{2} \Lambda,
$$

where $\Lambda$ is some energy UV cut-off. So first thing to note is that for $d<7$ (actually, for $d<6$ ) both the counterterm energy density and the fixed energy density only differ by a finite amount, so if one is sufficient to regulate the theory then, so is the other. Moreover, as most of our study corresponds to the fast quench regime where $m \delta t \ll 1$, this finite amount would be negligible compared to the scaling with $\delta t$ and so, the conclusion will be unchanged using either approach. ${ }^{9}$ Let us also note that the subtraction of $\mathcal{E}_{\text {fixed }}$ also satisfies the Ward identity. This is easy to see as the only time dependence is on the mass, so

$$
\partial_{t} \mathcal{E}_{\text {fixed }}=\partial_{t}\left(\sigma_{s}^{-1} \int d k k^{d-2} \sqrt{k^{2}+m^{2}(t)}\right)=\frac{1}{2}\left(\sigma_{s}^{-1} \int d k \frac{k^{d-2}}{\sqrt{k^{2}+m^{2}(t)}}\right) \partial_{t} m^{2}(t),
$$

but the term in parentheses in the final expression is just the bare expectation value of $\phi^{2}$ with an instantaneous mass $m(t)$ - compare to eq. (5.5) - , which gives exactly the Ward identity in eq. (2.12).

The situation is completely different in higher dimensions, though. The energy density of the quench has more divergences than those appearing in $\mathcal{E}_{\text {fixed }}$. These are proportional to time derivatives of the quenched coupling. If we suppose that all the quench happens within a time of order $\delta t$ of, say, $t=0$, then these terms will not affect what happens at very early and very late times, so it is possible to compute $\mathcal{E}_{\text {quench }}-\mathcal{E}_{\text {fixed }}$ in those regimes.

\footnotetext{
${ }^{9}$ See, however, the discussion on the reverse quench in $d=3$.
} 

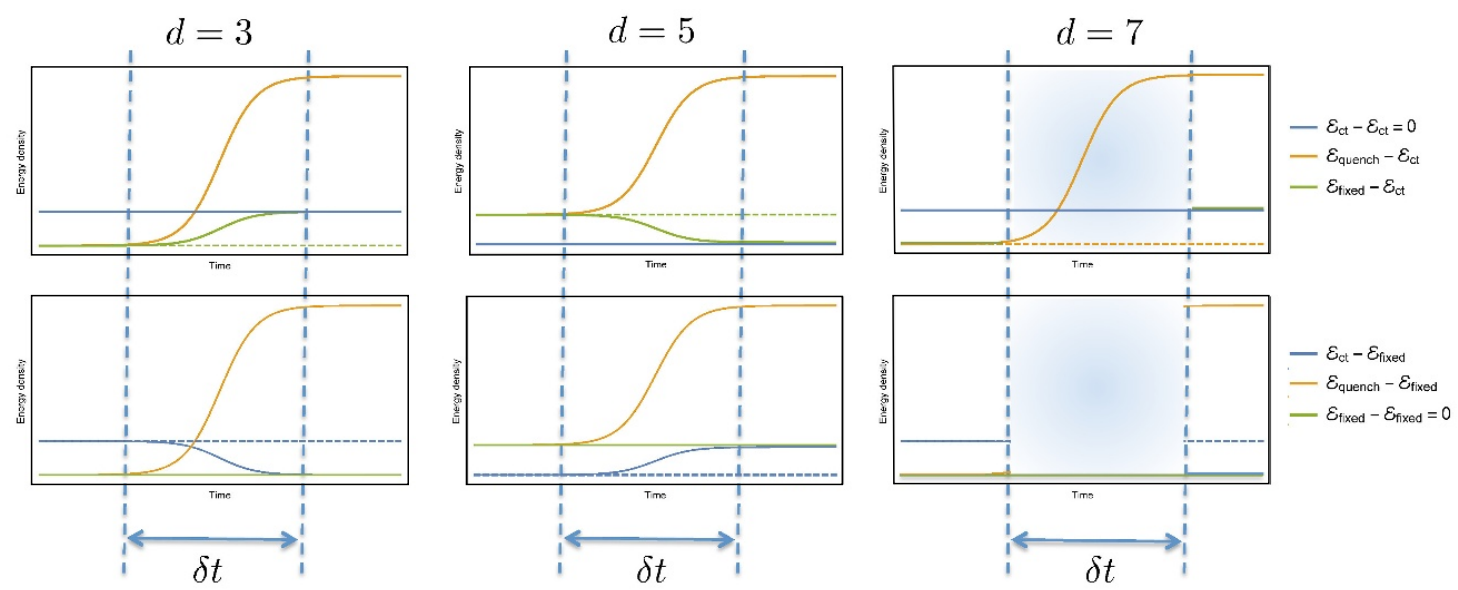

Figure 14. (Colour online) Schematic description of the evolution of the energy density after a quench as a function of time depending on the regularization scheme. In the first row we exemplify the counterterm subtraction that allows us to follow the evolution for any time, even during the quench itself, characterized by the time scale $\delta t$. Taking the counterterm energy density as the zero of energy density, at very early times, the starting energy density is negative for $d=4 k+3$ and positive for $d=4 k+1$, with $k \geq 0$ integer. In the second row we take the fixed energy density as the zero of energy density. In this case, all the quench energies are greater than this ground zero energy density. The reason is that the fixed mass energy density is the energy density for the scalar with a fixed mass and no quench, so any energy inserted during the quench will give a positive additional contribution. Note that in low dimensional spacetimes, the fixed energy density differs from the counterterm energy density but always by a finite amount proportional to a power of the mass at that instant of time, while the quench energy density usually scales also with $\delta t$, so our universal scalings, i.e., $\delta \mathcal{E} \sim \delta \lambda^{2} \delta t^{d-2 \Delta}$, will appear in any case. The same will happen in greater dimensions at very early and very late times. However, for $d \geq 6$ during the quench, it is not sufficient to subtract the fixed energy density, as there are extra UV divergences in the quenched energy density that are proportional to time derivatives of the mass. In this case, subtracting the fixed energy density is sufficient to compute the energy density at very early or very late times but not during the middle of the quench. This is depicted in the plot in the bottom-right corner for $d=7$ but is a general feature of higher dimensional spacetimes. In the same way, in the upper-right plot we cannot sketch $\mathcal{E}_{\text {fixed }}-\mathcal{E}_{c t}$ during the quench as they differ by an infinite amount.

However, if we want to follow the evolution through the actual quench, $\mathcal{E}_{\text {quench }}-\mathcal{E}_{\text {fixed }}$ is just divergent and we do not have a finite observable in the middle of the process. This is the main reason why $\mathcal{E}_{\text {ren }}$ is a better measure of what is going on during the quench, i.e., because it allows us to compute the energy density at any time in any spacetime dimensions.

All of these situations are depicted in figure 14, where to show clearly what is going on we set as the zero of energy density with $\mathcal{E}_{c t}$ in the first row and with $\mathcal{E}_{\text {fixed }}$ in the second. For $d=3$ and 5 , both approaches are valid and we see that the only difference is on some small quantity proportional to $m$. We showed both $d=3$ and $d=5$ because $\mathcal{E}_{\text {fixed }}$ and $\mathcal{E}_{c t}$ differ in each case by a different amount. While in $d=3$, the difference is negative, in $d=5$, it is positive. In $d=7$, however, we can follow $\mathcal{E}_{\text {ren }}$ but not the other one. Then, it is clear now that the naive intuition is wrong or at least is not complete. 
The next step would be to think whether there is some other way to regulate the energy density in higher dimensions. A possible answer already appeared in $[1,2]$ while not completely emphasized. We know that the counterterms come from an adiabatic expansion. At zeroth order, this adiabatic expansion gives just the fixed energy density that corresponds to doing the quench infinitely slowly so that, at each instant of time, the energy density is just the energy density needed for the scalar field to have that particular mass $m(t)$. What we do to get the counterterms in just to expand the adiabatic expansion for large momentum and then extract the divergent pieces. As mentioned, at zeroth order, the fixed energy density differs from the counterterm energy density by a finite piece proportional to $m^{d}(t)$. But we also know that for higher dimensional spacetimes we need to go to higher orders in the adiabatic expansion to capture the divergences involving time derivatives of the mass. So an idea to generalize the fixed energy density subtraction to higher dimensions would be that, instead of subtracting just the counterterms, to subtract the full energy density in the adiabatic expansion to that order. This would correspond to the energy of a slow quench but going beyond the zeroth order.

To be more explicit, in the adiabatic expansion shown in [2] we defined incoming modes of the form

$$
u_{\vec{k}}=\frac{1}{\sqrt{2 \Omega_{k}(t)}} \exp \left(i \vec{k} \cdot \vec{x}-i \int^{t} \Omega_{k}\left(t^{\prime}\right) d t^{\prime}\right) .
$$

Then we found that

$$
\Omega_{k}=\omega_{k}-\frac{1}{4 \omega_{k}}\left(\frac{\ddot{\omega}_{k}}{\omega_{k}}-\frac{3 \dot{\omega}_{k}^{2}}{2 \omega_{k}^{2}}\right)+O\left(\frac{1}{m^{3} \delta t^{4}}\right),
$$

where $\omega_{k}^{2}=k^{2}+m(t)^{2}$. So the zeroth order term in $\Omega_{k}$ is the fixed mode energy density but this is then corrected with a second term that is second order in time derivatives of the mass and so forth.

For $d=7$, we showed that it is enough to expand the energy density to that order. So instead of regulating the energy density with the counterterm energy density what we can do is to subtract, at any $t / \delta t$, the whole energy density coming from that second order expansion. This will include, of course, the necessary terms to cancel all the UV divergences but it will probably introduce some extra finite terms, in analogy to the extra finite piece that the fixed energy density has with respect to the counterterm energy density in lower dimensions. Let us add that this subtraction is also consistent with the Ward identity. ${ }^{10}$

${ }^{10}$ To see this one should use the $u_{\vec{k}}$ modes of eq. (8.4) to compute the energy density for the scalar field,

$$
\langle\mathcal{E}\rangle=\frac{\Omega_{d-2}}{2(2 \pi)^{d}} \int \frac{k^{d-2} d k}{2 \omega_{\text {in }}}\left(\left|\partial_{t} u_{\vec{k}}\right|^{2}+\left|\partial_{i} u_{\vec{k}}\right|^{2}+m(t)^{2}\left|u_{\vec{k}}\right|^{2}\right) .
$$

Upon taking the time derivative of that expression, one should get two different terms. First, a term proportional to

$$
\Omega_{k}^{2}-\omega_{k}^{2}-\frac{1}{2} \frac{\partial_{t}^{2} \Omega_{k}}{\Omega_{k}}+\frac{3}{4}\left(\frac{\partial_{t} \Omega_{k}}{\Omega_{k}}\right)^{2}
$$

that perfectly vanishes since that is the equation which $\Omega_{k}$ should satisfy in order for the modes to satisfy the equations of motion (see [2]). The second term, however, is not vanishing and gives exactly the Ward identity. 


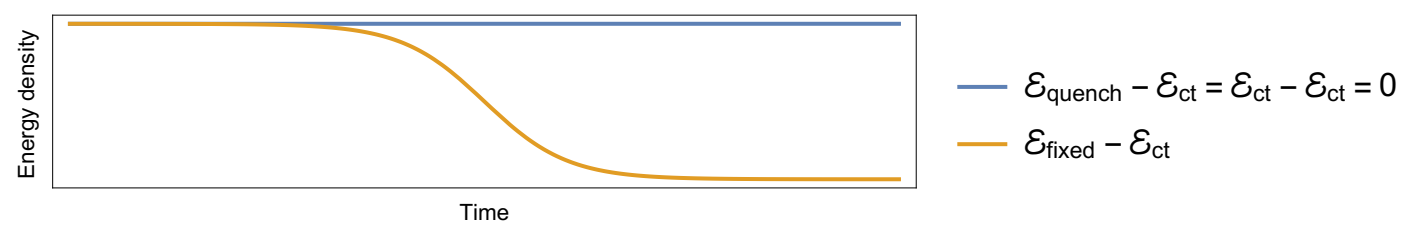

(a) The energy density using the counterterm subtraction.

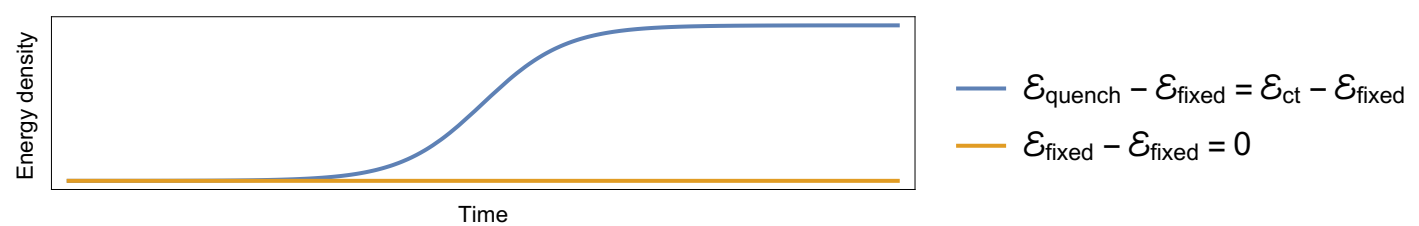

(b) The energy density using the fixed mass subtraction.

Figure 15. (Colour online) Schematic plots of the evolution of the energy density in three spacetime dimensions when $\delta t \rightarrow 0$. From the counterterm subtraction point of view, the work done is zero but from the fixed mass subtraction perspective, there is finite work done.

In all, we have two different consistent ways of regulating the energy density in our scalar field quenches. It would be interesting to consider whether there is some analogy of these two methods in holography. Our counterterm subtraction is clearly the counterpart of the holographic renormalization approach. But what would be the equivalent of subtracting the fixed mass energy density in an holographic setup? Well, it seems reminiscent of the old method of background subtraction in the early days of the AdS/CFT correspondence (see, for instance, [32]). Usually, divergences in holography appear as we take limits toward the boundary due to the divergent nature of pure anti-de Sitter spacetime as we take the radial coordinate towards the boundary. So, the first idea in holography, which was inherited from early semi-classical calculations in quantum gravity, to get a finite renormalized quantity for some excited state was to subtract that same quantity but in the vacuum state, i.e., in pure AdS. Then both quantities will be divergent but their subtraction would be finite and this is quite analogous to our fixed energy density subtraction. Later on, this procedure was replaced by the more rigorous method of holographic renormalization.

There is an interesting effect in the use of these two different approaches in $d=3$. In this case, the scaling is special because instead of giving a diverging behaviour as $\delta t \rightarrow 0$, it gives a vanishing one. We analysed this case in [2], concluding that actually the energy density produced was given by $\delta\langle\mathcal{E}\rangle_{\text {ren }}=\frac{m^{3}}{8 \pi}$, where $m$ is the initial mass. The interesting thing was that then, if we do the reverse quench where the initial mass is zero, it appears that the work done by the quench is zero! However, this was an artifact of using the counterterm energy density to regulate the expectation value. If we use the fixed energy density then we will find that the energy density starts from zero at early times and it goes to some finite value, giving some non-zero finite work in the process, as depicted in figure 15 .

Finally, we wish to consider one last method of obtaining a finite energy density. In section 6 , we considered the difference between two physical energies, i.e., $\mathcal{E}_{\text {quench }}-\mathcal{E}_{\text {ground }}$. Note that $\mathcal{E}_{\text {ground }}$, the ground-state energy density, should be something that we can easily define at very late and very early times. In particular, this looks like $\mathcal{E}_{\text {quench }}-\mathcal{E}_{\text {fixed }}$ at 
these early and late times. However, $\mathcal{E}_{\text {ground }}$ is a real physical energy density of a particular state and it can be defined in any renormalization scheme. So in this case, we do not need to make any reference to our choice of scheme because all of the divergences cancel in the difference of two physical energies. Of course, the drawback is that this can only be computed at very early or late times.

Even dimensions. Most of the explicit calculations presented in this paper refer to odd spacetime dimensions. However, most of the conclusions also hold for even dimensions. As pointed out in $[1,2]$, the only differences between even and odd dimensions are that there are additional logarithmic UV divergences which must be regulated in even dimensions, and as a result, the renormalized expectation values have an extra logarithmic enhancement in the $\delta t$ scaling. For example, in even dimensions, the expectation value for $\phi^{2}$ in the fast smooth quench scales as

$$
\left\langle\phi^{2}\right\rangle_{\mathrm{ren}} \sim \delta t^{4-d} \log \mu \delta t
$$

where $\mu$ is a new renormalization scale introduced by the logarithmic counterterms.

The appearance of logarithmic counterterms adds an additional technical difficulty to the calculations presented in this paper but we do not expect that they would change the main results. In particular, the renormalized expectation values of $\phi^{2}$ have a smooth limit at late times as $\delta t \rightarrow 0$ in higher even or odd dimensions, while the analogous quantity diverges after an instantaneous quench. With regards to the energy density, we presented results for lower even dimensions in section 6 , where we showed that the excess energy has a smooth limit in $d=2$ as $\delta t \rightarrow 0$, which matches the instantaneous answer. In higher dimensions, the excess energy diverges as expected from the scaling of $\left\langle\phi^{2}\right\rangle_{\text {ren }}$ and the Ward identity (2.12).

Lessons for interacting theories. We end this discussion with a comment on the lessons of our work for general interacting theories. In [1, 2] we showed that the scaling form of renormalized quantities holds for general quantum field theories for fast quenches as defined in eq. (1.1). It is natural to expect that the scaling for correlation functions found for the free theory in section 4 would have an analogue in interacting theories as well. When the length scale in the correlator is small compared to the quench time, this correlator can be viewed as a point-split version of the operator which is used for the quench and in the fast quench limit, the arguments of $[1,2]$ then show that this quantity would scale in the expected fashion.

In this paper, we found that the relationship between the fast limit of a smooth quench and an instantaneous quench is non trivial for free field theories in high dimensions. This again should generalize to interacting theories. What really led to the non trivial relation is the fact that in higher spacetime dimensions, the conformal dimension of the quenched operator becomes large. Indeed, the scaling of the renormalized quenched operator $\mathcal{O}$ for general interacting theory together with the Ward identity shows that the renormalized energy density at late times behaves as $\delta t^{d-2 \Delta}$ and therefore diverges as $\delta t \rightarrow 0$ for any $d$ whenever $\Delta>$ $d / 2$. This can be consistent with the results of an instantaneous quench only if the latter is UV divergent in this case. This fact should have non trivial consequences for the ability to 
express the state after a quench in terms of a boundary state as in $[10,11]$ even in low spacetime dimensions when the conformal dimension of the quenched operator is large enough.

\section{Acknowledgments}

We would like to thank Diptarka Das, Fabian Essler, Alfred Shapere, Stephen Shenker, Eva Silverstein and Lenny Susskind for discussions. S.R.D. would like to thank the Galileo Galilei Institute for Theoretical Physics for the hospitality and the INFN for partial support during the completion of this work. Research at Perimeter Institute is supported by the Government of Canada through Industry Canada and by the Province of Ontario through the Ministry of Research \& Innovation. RCM and DAG are also supported by an NSERC Discovery grant. RCM is also supported by research funding from the Canadian Institute for Advanced Research. The work of SRD is partially supported by the National Science Foundation grant NSF-PHY-1214341. DAG also thanks the Kavli Institute for Theoretical Physics for hospitality during the last stages of this project. Research at KITP is supported, in part, by the National Science Foundation under Grant No. NSF PHY11-25915.

\section{A Review of constant mass correlators}

In this appendix, we review the computation and behaviour of the (spatial) correlator for a massive free scalar field with a constant mass. For simplicity, we will focus on odd spacetime dimensions. Hence we are interested in computing the following spatial correlator,

$$
C(\vec{r}) \equiv\langle\phi(\vec{r}) \phi(\overrightarrow{0})\rangle=\frac{1}{2(2 \pi)^{d-1}} \int \frac{d^{d-1} k}{\sqrt{k^{2}+m^{2}}} e^{i \vec{k} \cdot \vec{r}},
$$

where $m$ is simply a fixed constant (for all time). First, we can choose, without loss of generality, to place $\vec{r}$ along one particular axis using the rotational symmetry of the problem. Integrating out the transverse angular directions, then yields

$$
C(\vec{r})=\frac{\Omega_{d-3}}{2(2 \pi)^{d-1}} \int \frac{k^{d-2} d k}{\sqrt{k^{2}+m^{2}}} \int_{0}^{\pi} d \theta \sin ^{d-3} \theta e^{i k r \cos \theta}
$$

where $k=|\vec{k}|$ and $r=|\vec{r}|$. The integral over $\theta$ can be done analytically and for odd $d$, we find

$$
C(\vec{r})=\frac{1}{\sigma_{c} r^{\frac{d-3}{2}}} \int \frac{k^{\frac{d-1}{2}} d k}{\sqrt{k^{2}+m^{2}}} J_{\frac{d-3}{2}}(k r),
$$

where $\sigma_{c}=2^{\frac{d+1}{2}} \pi^{\frac{d-1}{2}}$ and $J_{\frac{d-3}{2}}$ is the Bessel function of order $\frac{d-3}{2}$.

Now, to get the full answer for $C(\vec{r})$ we need to integrate over all $k$, so we note that for large $k$, the Bessel function behaves as $1 / \sqrt{k r}$ times some linear combination of trigonometric functions (of $k r$ ). Hence the naïve counting of the powers of $k$ would yield an overall factor of $k^{\frac{d-4}{2}}$ in the integrand above and hence one might conclude that the integral would diverge for any $d(\geq 2)$. However, this factor provides a envelope for a rapidly oscillating function which tends to produce an added cancellation in the integral. 
Integrals of this form can be defined with the following regulator: insert an additional factor of the form $\exp (-a k)$ to the desired integrand. The resulting (finite) answer now remains finite in the limit of $a \rightarrow 0$. In fact, one can show that this method works correctly for integrands that are a product of some power of $k$ times an oscillatory function around zero. For example, one can show that,

$$
\begin{aligned}
\int_{0}^{\infty} d k k^{\alpha} \sin (x k+\delta) & \equiv \lim _{a \rightarrow 0} \int_{0}^{\infty} d k k^{\alpha} \sin (x k+\delta) \exp (-a k) \\
& =\sin \left(\frac{\pi}{2}(\alpha+1)+\delta\right) \Gamma(\alpha+1) x^{-(\alpha+1)}
\end{aligned}
$$

for any non-negative values of $\alpha$ and $x$. Applying this apprach to eq. (A.3) yields the following analytic answer

$$
C(\vec{r})=\frac{1}{(2 \pi)^{d / 2}}\left(\frac{m}{r}\right)^{\frac{d-2}{2}} K_{1-\frac{d}{2}}(m r),
$$

where now $K_{\alpha}$ is the Bessel $\mathrm{K}$ function. Of course, one can readily verify that this correlator satisfies the Klein-Gordon equation as desired. ${ }^{11}$

Given this expression (A.5), it is straightforward to examine various asymptotics of the correlator. In particular, considering the limit $m r \rightarrow 0$, we obtain

$$
\begin{aligned}
\sigma_{c} C(\vec{r})= & \frac{\Gamma\left(\frac{d}{2}-1\right) 2^{\frac{d-3}{2}}}{\sqrt{\pi} r^{d-2}}\left(1-\frac{m^{2} r^{2}}{2(d-4)}+\frac{m^{4} r^{4}}{8(d-4)(d-6)}+O\left(m^{6} r^{6}\right)\right)+ \\
& +\frac{\Gamma\left(1-\frac{d}{2}\right)}{\sqrt{\pi} 2^{\frac{d-1}{2}}} m^{d-2}+O\left(m^{d} r^{2}\right) .
\end{aligned}
$$

Hence the correlator diverges as expected as $r \rightarrow 0$, i.e., the leading divergence goes as $1 / r^{d-2}$. The above expansion reveals that the subleading terms are all proportional to the mass in this limit. To have some concrete examples, we show:

$$
\begin{array}{ll}
d=3, & \sigma_{c} C(\vec{r})=\frac{1}{r}-m+O\left(m^{2} r\right), \\
d=5, & \sigma_{c} C(\vec{r})=\frac{1}{r^{3}}-\frac{m^{2}}{2 r}+\frac{m^{3}}{3}+O\left(m^{4} r\right), \\
d=7, & \sigma_{c} C(\vec{r})=\frac{3}{r^{5}}-\frac{m^{2}}{2 r^{3}}+\frac{m^{4}}{8 r}-\frac{m^{5}}{15}+O\left(m^{6} r\right) .
\end{array}
$$

We also note that the leading term in eq. (A.6) is, in fact, the exact answer for the massless correlator, i.e.,

$$
C(\vec{r})=\frac{\Gamma\left(\frac{d-2}{2}\right)}{4 \pi^{\frac{d}{2}}} \frac{1}{r^{d-2}} \quad \text { for } m=0 .
$$

The other interesting limit to consider is $m r \rightarrow \infty$. In this case, the Bessel function decays exponentially and we find

$$
C(\vec{r})=\frac{e^{-m r}}{\sigma_{c}} \frac{m^{\frac{d-3}{2}}}{r^{\frac{d-1}{2}}}(1+O(1 /(m r))) .
$$

\footnotetext{
${ }^{11}$ The correlator (A.5) applies for general spacelike separations if we replace the distance $r$ by $\sqrt{r^{2}-t^{2}}$.
} 
We might note that the power of $r$ in the leading term in eqs. (A.6) and (A.9) happens to coincide for $d=3$ but otherwise they differ.

To conclude this appendix, we emphasize the two main results: the first one is that naïvely the integrals above seem to be divergent, especially for high $d$. However, because the integrand is mainly oscillating around zero, they can be regulated as in eq. (A.4) to get a finite result. The second lesson is that in the static case this correlator diverges as $1 / r^{d-2}$, as shown in eqs. (A.6) and (A.7). We will take these facts into account when we analyse spatial correlators in the instantaneous and smooth quenches in section 3.

Open Access. This article is distributed under the terms of the Creative Commons Attribution License (CC-BY 4.0), which permits any use, distribution and reproduction in any medium, provided the original author(s) and source are credited.

\section{References}

[1] S.R. Das, D.A. Galante and R.C. Myers, Universal scaling in fast quantum quenches in conformal field theories, Phys. Rev. Lett. 112 (2014) 171601 [arXiv:1401.0560] [InSPIRE].

[2] S.R. Das, D.A. Galante and R.C. Myers, Universality in fast quantum quenches, JHEP 02 (2015) 167 [arXiv:1411.7710] [INSPIRE].

[3] S. Mondal, D. Sen and K. Sengupta, Non-equilibrium dynamics of quantum systems: order parameter evolution, defect generation, and qubit transfer, in Quantum Quenching, Anealing and Computation, Lect. Notes Phys. 802 (2010) 21 [arXiv:0908.2922].

[4] J. Dziarmaga, Dynamics of a quantum phase transition and relaxation to a steady state, Adv. Phys. 59 (2010) 1063 [arXiv:0912.4034].

[5] A. Polkovnikov, K. Sengupta, A. Silva and M. Vengalattore, Nonequilibrium dynamics of closed interacting quantum systems, Rev. Mod. Phys. 83 (2011) 863 [arXiv:1007.5331] [INSPIRE].

[6] A. Lamacraft and J.E. Moore, Potential insights into non-equilibrium behavior from atomic physics, in Ultracold Bosonic and Fermionic Gases, A. Fletcher, K. Levin and D. Stamper-Kurn eds., Contemporary Concepts in Condensed Matter Science, Elsevier, The Netherlands (2012) [arXiv:1106.3567].

[7] T.W.B. Kibble, Topology of cosmic domains and strings, J. Phys. A 9 (1976) 1387 [inSPIRE].

[8] W.H. Zurek, Cosmological experiments in superfluid helium?, Nature 317 (1985) 505 [INSPIRE].

[9] A. Chandran, A. Erez, S.S. Gubser and S.L. Sondhi, Kibble-Zurek problem: universality and the scaling limit, Phys. Rev. B 86 (2012) 064304 [arXiv:1202.5277].

[10] P. Calabrese and J.L. Cardy, Time-dependence of correlation functions following a quantum quench, Phys. Rev. Lett. 96 (2006) 136801 [cond-mat/0601225] [INSPIRE].

[11] P. Calabrese and J. Cardy, Quantum quenches in extended systems, J. Stat. Mech. (2007) P06008 [arXiv: 0704.1880] [INSPIRE].

[12] S. Sotiriadis and J. Cardy, Quantum quench in interacting field theory: a self-consistent approximation, Phys. Rev. B 81 (2010) 134305 [arXiv: 1002.0167] [INSPIRE]. 
[13] V. Gritsev and A. Polkovnikov, Universal dynamics near quantum critical points, arXiv:0910.3692 [INSPIRE].

[14] P. Basu and S.R. Das, Quantum quench across a holographic critical point, JHEP 01 (2012) 103 [arXiv: 1109.3909] [INSPIRE].

[15] P. Basu, D. Das, S.R. Das and T. Nishioka, Quantum quench across a zero temperature holographic superfluid transition, JHEP 03 (2013) 146 [arXiv:1211.7076] [INSPIRE].

[16] P. Basu, D. Das, S.R. Das and K. Sengupta, Quantum quench and double trace couplings, JHEP 12 (2013) 070 [arXiv: 1308.4061] [InSPIRE].

[17] J. Sonner, A. del Campo and W.H. Zurek, Universal far-from-equilibrium dynamics of a holographic superconductor, arXiv:1406.2329 [INSPIRE].

[18] P.M. Chesler, A.M. Garcia-Garcia and H. Liu, Defect formation beyond Kibble-Zurek mechanism and holography, Phys. Rev. X 5 (2015) 021015 [arXiv:1407.1862] [INSPIRE].

[19] S.R. Das and T. Morita, Kibble-Zurek scaling in holographic quantum quench: backreaction, JHEP 01 (2015) 084 [arXiv: 1409.7361] [INSPIRE].

[20] K. Murata, S. Kinoshita and N. Tanahashi, Non-equilibrium condensation process in a holographic superconductor, JHEP 07 (2010) 050 [arXiv: 1005.0633] [INSPIRE].

[21] M.J. Bhaseen, J.P. Gauntlett, B.D. Simons, J. Sonner and T. Wiseman, Holographic superfluids and the dynamics of symmetry breaking, Phys. Rev. Lett. 110 (2013) 015301 [arXiv: 1207.4194] [INSPIRE].

[22] A.M. García-García, H.B. Zeng and H.Q. Zhang, A thermal quench induces spatial inhomogeneities in a holographic superconductor, JHEP 07 (2014) 096 [arXiv:1308.5398] [INSPIRE].

[23] A. Buchel, L. Lehner and R.C. Myers, Thermal quenches in $N=2 *$ plasmas, JHEP 08 (2012) 049 [arXiv:1206.6785] [INSPIRE].

[24] A. Buchel, L. Lehner, R.C. Myers and A. van Niekerk, Quantum quenches of holographic plasmas, JHEP 05 (2013) 067 [arXiv: 1302.2924] [INSPIRE].

[25] A. Buchel, R.C. Myers and A. van Niekerk, Universality of abrupt holographic quenches, Phys. Rev. Lett. 111 (2013) 201602 [arXiv:1307.4740] [INSPIRE].

[26] D. Berenstein and A. Miller, Conformal perturbation theory, dimensional regularization and AdS/CFT correspondence, Phys. Rev. D 90 (2014) 086011 [arXiv:1406.4142] [INSPIRE].

[27] N.D. Birrell and P.C.W. Davies, Quantum fields in curved space, Cambridge University Press, Cambridge U.K. (1982).

[28] S.R. Das, D.A. Galante and R.C. Myers, in preparation.

[29] M. Henningson and K. Skenderis, The holographic Weyl anomaly, JHEP 07 (1998) 023 [hep-th/9806087] [INSPIRE].

[30] V. Balasubramanian and P. Kraus, A stress tensor for anti-de Sitter gravity, Commun. Math. Phys. 208 (1999) 413 [hep-th/9902121] [INSPIRE].

[31] R. Emparan, C.V. Johnson and R.C. Myers, Surface terms as counterterms in the AdS/CFT correspondence, Phys. Rev. D 60 (1999) 104001 [hep-th/9903238] [INSPIRE].

[32] S.S. Gubser, I.R. Klebanov and A.A. Tseytlin, Coupling constant dependence in the thermodynamics of $N=4$ supersymmetric Yang-Mills theory, Nucl. Phys. B 534 (1998) 202 [hep-th/9805156] [INSPIRE]. 